\title{
Baicalin alleviates hyperglycemia-induced endothelial impairment via Nrf2
}

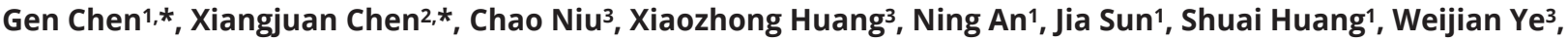 \\ Santie Li1, Yingjie Shen'1, Jiaojiao Liang1, Weitao Cong1 and Litai Jin'1 \\ 1School of Pharmaceutical Science, Wenzhou Medical University, Wenzhou, China \\ 2Department of Obstetrics and Gynecology, the First Affiliated Hospital of Wenzhou Medical University, Wenzhou, China \\ ${ }^{3}$ The Second Affiliated Hospital and Yuying Children's Hospital of Wenzhou Medical University, Wenzhou, China \\ Correspondence should be addressed to L Jin: jin_litai@126.com \\ *(G Chen and X Chen contributed equally to this work)
}

\begin{abstract}
Baicalin is the major component found in Scutellaria baicalensis root, a widely used herb in traditional Chinese medicine, which exhibits strong anti-inflammatory, anti-viral and anti-tumor activities. The present work was devoted to elucidate the molecular and cellular mechanisms underlying the protective effects of Baicalin against diabetesinduced oxidative damage, inflammation and endothelial dysfunction. Diabetic mice, induced by streptozotocin (STZ), were treated with intraperitoneal Baicalin injections. Human umbilical vein endothelial cells (HUVECs) were cultured either in normal glucose (NG, $5.5 \mathrm{mM}$ ) or high glucose (HG, $33 \mathrm{mM})$ medium in the presence or absence of Baicalin for $72 \mathrm{~h}$. We observed an obvious inhibition of hyperglycemia-triggered oxidative damage and inflammation in HUVECs and diabetic aortal vasculature by Baicalin, along with restoration of hyperglycemia-impaired nuclear factor (erythroidderived 2)-like 2 (Nrf2) pathway activity. However, the protective effects of Baicalin almost completely abolished in HUVECs transduced with shRNA against Nrf2, but not with nonsense shRNA. Mechanistic studies demonstrated that HG decreased Akt and GSK3B phosphorylation, restrained nuclear export of Fyn and nuclear localization of Nrf2, blunted Nrf2 downstream target genes and subsequently induced oxidative stress in HUVECs. However, those destructive cascades were well prevented by Baicalin in HUVECs. Furthermore, LY294002 and ML385 (inhibitor of PI3K and Nrf2) attenuated Baicalin-mediated Nrf2 activation and the ability of facilitates angiogenesis in vivo and ex vivo. Taken together, the endothelial protective effect of Baicalin under hyperglycemia condition could be partly attributed to its role in downregulating reactive oxygen species (ROS) and inflammation via the Akt/GSK3B/Fyn-mediated Nrf2 activation.
\end{abstract}

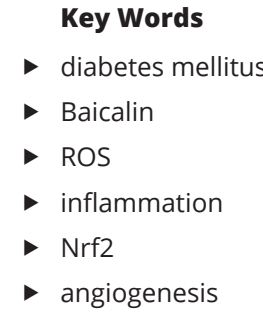

Journal of Endocrinology (2019) 240, 81-98

\section{Introduction}

Diabetes is one of the greatest global health emergencies of the 21st century. In 2017, 415 million adults had diabetes, and this number is estimated to increase to 693 million in 2045 (Cho et al. 2018). Vascular complications in diabetes are considered as major causes for shortened life expectancy and high mortality rate (Grundy et al. 2002). Moreover, endothelial dysfunction, which causes defective endothelial repair and inability of angiogenesis, 
is also recognized as an important causative factor in the pathogenesis of diabetes-associated cardiovascular disease (Erusalimsky 2009).

Studies in experimental animal models and cultured cells have demonstrated that oxidative stress is a major contributor to the diabetic vascular complication and increased ROS largely accounts for this dysfunction (Guzik et al. 2002, Bhatt et al. 2016). Alterations of ROS levels may include superoxide anions, hydrogen peroxide and hydroxyl radicals, which result in impaired homeostasis and associated pathologies (D'Autreaux \& Toledano 2007, Pitocco et al. 2013). In addition, ROS stimulates the activation of NF- $\mathrm{kB}$ proteins (Vallée \& Lecarpentier 2018), which plays a critical regulating role in the release of pro-inflammatory cytokines such as IL6, IL8 and TNFa (Tang et al. 2017). Therefore, protecting vascular cells from oxidative stress injury is a major approach in the treatment of diabetic complications (Rask-Madsen \& King 2013).

Nrf2 is one of the most important cellular defense mechanisms to cope with oxidative stress (Itoh et al. 1999). It regulates intracellular antioxidants, phase II detoxifying enzymes and many other proteins that detoxify xenobiotics and neutralize ROS to promote cell survival and maintain cellular redox homeostasis (Zhang 2006). NAD(P)H dehydrogenase quinone 1, 2 (NQO1, NQO2), superoxide dismutase 2 (SOD2), heme oxygenase 1 (HO1) and catalase (CAT) are among the well-studied Nrf2 target genes that can be upregulated through binding to the antioxidant response element and then initiates the transcription of an array of genes in response to oxidative stress (Chan et al. 2001). Recently, the essential role of Nrf2 in protecting against diabetic vascular diseases has been reported (Xue et al. 2008, Li et al. 2011, Palsamy \& Subramanian 2011). It seems likely that Nrf2-mediated anti-oxidative capacity could act to counterbalance the stress induced by increased ROS production. Furthermore, activation of Nrf2 has been shown to be protective against hyperglycemia-induced apoptosis and cell damage in renal, cardiac and vascular cells (Li et al. 2012). Hence, Nrf2 signaling represents a therapeutic target for cardiovascular complications in obesity and diabetes.

Flavonoids, a widely distributed bioactive component in plant, possess antioxidant potential both in vivo and in vitro by scavenging ROS (Lin et al. 2005). Therefore, flavonoids are considered therapeutic remedies for diabetic complications (Halliwell et al. 2005, Williamson et al. 2005). Baicalin (5,6,7-trihydroxyflavone-7- $\beta$-Dglucuronide) is one of the principal flavonoid isolated from the dried root of Scutellaria baicalensis Georgi (Li et al. 2011). Although Baicalin is generally low-toxic to normal tissues, it exhibits strong anti-inflammatory, antiviral and anti-tumor activities (Srinivas 2010). Baicalin has recently been reported to exhibit anti-hyperglycemic effects in STZ-induced diabetic Wistar rats (Li et al. 2011). In addition, Baicalin was reported to ameliorate $\mathrm{H}_{2} \mathrm{O}_{2}$ induced cytotoxicity, reduce oxidative stress and ER stress and further activate the anti-oxidative Nrf2 signaling pathway (Lin et al. 2014). Nevertheless, the precise molecular mechanisms by which hyperglycemia cause a wide variety of vascular complications and cardiovascular dysfunction are not yet well understood. Therefore, the aims of the present study are to determine whether Baicalin ameliorate hyperglycemia-induced endothelial dysfunction via activating Nrf2-mediated exogenous antioxidant and to explore its related signal pathway.

\section{Materials and methods}

\section{Animals}

Male C57BL/ 6 mice (6-8 weeks, 18 to $23 \mathrm{~g}$ ), were purchased from the Model Animal Research Center of Nanjing University (Nanjing, China). The mice were housed in an environmentally controlled room for 4-6 days to adapt to the environment before experimentation. Diabetes mellitus was induced in 8-week-old male mice by intraperitoneally (i.p.) injecting $100 \mathrm{mg} / \mathrm{kg}$ STZ (V900890, Sigma-Aldrich; Lu et al. 2012). Then the diabetic mice were divided into four groups and subjected to the following treatment regimens: (1) ad libitum feeding of chow diets; (2) ad libitum feeding of chow diets and vehicle injection of dimethyl sulfoxide (DMSO; D8418, Sigma-Aldrich); (3) ad libitum feeding of chow diets and i.p. injections of $50 \mathrm{mg} / \mathrm{kg} /$ day Baicalin (572667, Sigma-Aldrich; Chen et al. 2012) and (4) ad libitum feeding of chow diets and i.p. injections of $50 \mathrm{mg} / \mathrm{kg} /$ day Baicalin in the presence of $30 \mathrm{mg} / \mathrm{kg} /$ day ML385 (SML1833, Sigma-Aldrich; Singh et al. 2016) or $5 \mathrm{mg} / \mathrm{kg} /$ day LY294002 (L9908, Sigma-Aldrich; Li et al. 2013). After a 4-week course of treatment, corresponding analyses were performed. All male C57BL/6J mice were kept in a standard laboratory condition of temperature $21 \pm 2^{\circ} \mathrm{C}$, relative humidity $50 \pm 15 \%, 12 \mathrm{~h}$ light-darkness cycles, with water and food available ad libitum. All animal experiments and methods performed in this study followed ethical guidelines for animal studies and were approved by the Institutional Animal Care and Use Committee of Wenzhou Medical University, China. 


\section{Cell culture}

Human umbilical vein endothelial cells (HUVECs) and human aortal endothelial cells (HAOECs) were purchased from Lonza, Basel, Switzerland and cultured in endothelial cell growth medium-2(EGM-2, CC-3156\& CC-4176, Lonza) at $37^{\circ} \mathrm{C}$ in a $5 \% \mathrm{CO}_{2}$ humidified incubator until the start of experiment. Five to seven passage subconfluent cells were used in the experiments. Before starting the experimental procedures, the medium was removed and replaced with phenol red-free low-glucose DMEM (11054020, Gibco BRL) supplemented with 1\% calf serum (16010159, Gibco BRL) for $12 \mathrm{~h}$, and then endothelial cells were placed in EGM-2 consisting of either NG $(5.5 \mathrm{mM})$ or HG $(33 \mathrm{mM})$ in the presence or absence of Baicalin $(50 \mu \mathrm{M})$ for $72 \mathrm{~h}$, mannitol (MAN, $33 \mathrm{mM}$ : $5.5 \mathrm{mM}$ of glucose $+27.5 \mathrm{mM}$ of D-mannitol; M4125, Sigma-Aldrich) was served as the osmotic control for the HG, pharmacological antioxidant molecules N-Acetyl-L-cysteine (NAC, $2 \mathrm{mM}$; V900429, Sigma-Aldrich) was pretreated for $2 \mathrm{~h}$ every day to evaluate the effect of Baicalin on the oxidative stress. Media were changed every $24 \mathrm{~h}$. For signaling pathway analysis, each pathway antagonist: LY294002 $(10 \mu \mathrm{M})$ and ML385 $(20 \mu \mathrm{M})$ were pretreated for $2 \mathrm{~h}$ every day before Baicalin administration. All described experiments were independently carried out at least 3-6 separate times, with endothelial cells used between passages 3 to 20 .

\section{Adenovirus vector construction, virus production and infection}

Recombinant adenovirus vectors were constructed, propagated and titered as previously described (Maejima et al. 2013). Briefly, pBHGlox $\Delta \mathrm{E} 1,3$ Cre plasmid (Microbix, PD-01-40), including the $\Delta \mathrm{E} 1$ adenoviral genome, was co-transfected with the pDC316 shuttle vector containing the gene of interest into HEK293 cells using Lipofectamine 2000 (Life Technologies, 11668019). Through homologous recombination, the test genes were integrated into the E1-deleted adenoviral genome. The viruses were propagated in HEK293 cells.

The pSilencer 2.1-U6 expression vector was purchased from Ambion (Ambion, AM5762). The U6 RNA polymerase III promoter and the polylinker region were subcloned into the adenoviral shuttle vector pDC311 (Microbix, PD-01-25). The Nrf2 shRNA targeting sequence was 5'-GCAGTTCAATGAAGCTCAACT-3'. Sh-Scramble, an in-house generated shRNA adenovirus that encodes a scramble sequence 5'-TTCTCCGAACGTGTCACGT-3', was used as control. Recombinant adenoviruses were generated by homologous recombination in HEK293 cells as described above.

To knockdown Nrf2 expression in late HUVECs, HUVECs were infected with the adenoviral containing shRNA against Nrf2 or nonsense shRNA overnight and the medium was replaced with fresh growth medium $24 \mathrm{~h}$ after infection. After transfection for $48 \mathrm{~h}$, the levels of Nrf2 expression were detected by Western blot.

\section{TUNEL staining}

The cells were then stained with an In Situ Cell Death Detection Kit (Roche, 11684795910) according to the manufacturer's protocol. The stained cells were imaged with a confocal laser scanning microscope (TCS SP8, Leica). One hundred cells per field were counted and the percentage of TUNEL-positive cells was calculated.

\section{RNA isolation and semi-quantitative RT-PCR (sqRT-PCR)}

Total RNA was extracted from HUVECs by using TRIzol Reagent (15596018, Invitrogen) according to the

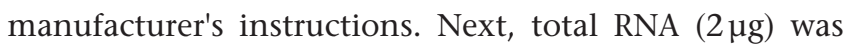
reverse transcribed into cDNA by using GoScript Reverse Transcription Kit (A5001, Promega). The cDNA was then subjected to sqRT-PCR analysis, and gene expression was quantified as previously described (Magalhães et al. 2013). The NQO1, NQO2, HO1, SOD2, CAT, IL1B, IL6, IL8 and TNFa amplification signals were normalized to glyceraldehyde-3-phosphate dehydrogenase (GAPDH) expression. Gene-specific primer sequences used for sqRT-PCR are listed in Table 1.

\section{Western blot}

Briefly, $30 \mu \mathrm{g}$ protein from each sample was resolved by SDS-PAGE on Tris-Glycine gels, and transferred to PVDF membrane. Membranes were blocked with 5\% (v/v) bovine serum albumin in Tris-buffered saline containing $0.1 \%(\mathrm{v} / \mathrm{v})$ Tween 20 (TBST) and incubated with primary antibodies overnight at $4^{\circ} \mathrm{C}$. Membranes were washed three times for $5 \mathrm{~min}$ with TBST, incubated in either HRPgoat-anti-mouse (ab6789, Abcam) or HRP-goat-anti-rabbit (ab6721, Abcam) secondary antibodies for $1 \mathrm{~h}$ at room temperature. Immunoreactive bands were visualized using Pierce ECL plus Western blotting substrate (32132, Thermo Fisher Scientific).

For nuclear Nrf2 accumulation assays, HUVECs and HAOECs were harvested and lysed to obtain cytoplasmic 
Table 1

\begin{tabular}{ll}
\hline NQO1 & GAAGAGCACTGATCGTACTGGC \\
& GGATACTGAAATTCGCAGGG \\
NQ2 & GTACTCATTGTCTATGCACACCA \\
& TGCCTGCTCAGTTCATCTACA \\
HO1 & GCCCCAGGATTTGCAGAGG \\
& GGAGGCCATCACCAGCTTGAA \\
SOD2 & GTCACCGAGGAGAGTACCAGGAGGCG \\
& CGCCTCCTGGTACTTCTCCTCGGTGAC \\
CAT & CCTTCTTGTTCAGGATGTGGTTTC \\
& CATGTGTGACCTCAAAGTAGCCAAA \\
IL1B & TGGGAGTAGATAAGGTACAGCCC \\
& GTAGTGGTGGTCGGAGATTCG \\
IL6 & ACTCACCTCTTCAGAACGAATTG \\
& CCATCTTTGGAAGGTTCAGGTTG \\
IL8 & GCCAACACAGAAATTATTGTAAAGCTT \\
& AATTCTCAGCCCTCTTCAAAAACTT \\
TNFa & CATCTTCTCAAAACTCGAGTACAA \\
& TGGGAGTAGATAAGGTACAGCCC \\
GAPDH & ATGGTGAAGGTCGGGTGAA \\
& TGGAAGATGGTGATGGGCTT \\
\hline
\end{tabular}

and nuclear lysates using the Keygen Nuclear-Cytosol Protein Extraction Kit from Nanjing KeyGen Biotech. Co., Ltd. (Nanjing, China).

The primary antibodies used to probe the membranes included Nrf2 (1:1000), Fyn (1:2000) and GAPDH (1:5000) were purchased from Santa Cruz Biotechnology, Santa Cruz; Akt and p-Akt (Ser473; 1:2000), GSK3B and p-GSK3B (Ser9; 1:2000), c-Caspase 3 (1:1000) were purchased from Cell Signaling Technology; 3-nitrotyrosine (1:2000), Bax (1:2000) and Bcl-2 (1:2000) were purchased from Abcam. The expression of specific antigens was quantified using ImageQuant 5.2 software (Molecular Dynamics), and the expression of GAPDH was used as loading control.

\section{In vitro angiogenesis (Tube formation) assay}

The in vitro angiogenic activity of HUVECs was determined by Matrigel tube formation assay. After the experimental period described above, the HUVECs were stained with cell-permeable dye, calcein (354216, Corning incorporated, Corning), for $30 \mathrm{~min}$ and replated in 24-well plates precoated with $150 \mu \mathrm{L} /$ well growth factor-reduced Matrigel (354234, Corning incorporated) and incubated at $37^{\circ} \mathrm{C}$ in cell culture incubator. After $12 \mathrm{~h}$ of incubation, capillary-like tube formation was observed with a computer-assisted microscope (EVOS, Thermo Fisher Scientific). Tube formation was defined as a tubelike structure exhibiting a length four times its width. The tube length in duplicate wells was counted and averaged using ImageJ software.

\section{Quantitative determination of oxidative stress (DHE assay)}

To detect the ROS level of HUVECs with HG treatment, dihydroethidium (DHE, D7008, Sigma-Aldrich) probe was used to stain HUVECs. DHE is cell permeable and able to react with superoxide to form ethidium, which in turn intercalates with DNA and produces nuclear fluorescence. HUVECs were seeded on 24-well plates and treated with $\mathrm{HG}$ in presence or absence Baicalin for $72 \mathrm{~h}$ and then incubated with $5 \mu \mathrm{M}$ DHE in DMSO for $30 \mathrm{~min}$ at $37^{\circ} \mathrm{C}$. Nuclear DHE-positive staining indicates superoxide generation in cells. The fluorescence intensity was observed with a computer-assisted microscope (EVOS, Thermo Fisher Scientific).

\section{Aortic ring assays}

To establish a direct action of Baicalin on vascular, thoracic aortae from 8-week-old C57/BL6 mice were surgically isolated, cleaned and dissected into $0.5 \mathrm{~mm}$ rings. Rings were embedded in $1 \mathrm{mg} / \mathrm{mL}$ of type I collagen (Millipore, 08-115) in a 96-well plate as described previously (Aplin et al. 2008, Baker et al. 2011). When embedded, the rings were cultured in serum-free endothelial basal medium (EBM) (CC-3121, Lonza) consisting of either NG (5.5 mM) or $\mathrm{HG}(33 \mathrm{mM})$ in the presence or absence of Baicalin $(50 \mu \mathrm{M})$, MAN $(33 \mathrm{mM}: 5.5 \mathrm{mM}$ of glucose $+27.5 \mathrm{mM}$ of D-mannitol) was served as the osmotic control for the HG. For signaling pathway analysis, each pathway antagonist: LY294002 $(10 \mu \mathrm{M})$ or ML385 $(20 \mu \mathrm{M})$ was pretreated for $2 \mathrm{~h}$ every day before Baicalin administration. Endothelial microvessel sprouts growing out from the rings were counted during the exponential growth phase to obtain angiogenic response data. Before the regression phase, rings were fixed with $4 \%(\mathrm{w} / \mathrm{v})$ paraformaldehyde for $30 \mathrm{~min}$. Pictures were taken on day 12 with a computerassisted microscope (Eclipse Ni, NIKON), and the total number of branches was counted using ImageJ (National Institutes of Health).

\section{Immunofluorescence staining}

For quantification of CD31 or 3-NT positive area, immunofluorescence staining was performed within the aortic wall. Eight micrometer paraffin sections were cut and incubated with anti-CD31 (ab24590, Abcam) or anti3-NT (ab61392, Abcam). After washing, samples were incubated with Alexa Fluor 647-conjugated anti-rabbit 
IgG secondary antibody (ab150115, Abcam) at a dilution of 1:200 for $60 \mathrm{~min}$ at room temperature. Cell nuclei were labeled by DAPI. The stained aortic walls were imaged with a confocal laser scanning microscope (TCS SP8, Leica). The total tissue area and the CD31 or 3-NT-stained positive area were measured with ImageJ software. Data were expressed as percentage of positive staining area per analyzed area.

For quantification of nuclear localization of Nrf2 in HUVECs. Briefly, cultured cells in six-well plates were fixed with $4 \%(\mathrm{w} / \mathrm{v})$ paraformaldehyde for $30 \mathrm{~min}$ and permeabilized with $0.5 \%(\mathrm{v} / \mathrm{v})$ Triton-X 100 (Solabio, Beijing, China) for $10 \mathrm{~min}$ at $37^{\circ} \mathrm{C}$. After wash, the cells were blocked in $5 \%(\mathrm{v} / \mathrm{v}) \mathrm{BSA}$ for $2 \mathrm{~h}$ and incubated with primary rabbit antibody against Nrf2 (1:200, Santa Cruz Biotechnology) at $4^{\circ} \mathrm{C}$ overnight. After incubation and wash, cells were blocked with Alexa Fluor 488-conjugated anti-mouse IgG secondary antibody (ab150113, Abcam) for $1 \mathrm{~h}$. After washes with PBS, the cell nuclei were stained with DAPI for $15 \mathrm{~min}$. Photo capture was performed by a confocal laser scanning microscope (TCS SP8, Leica). Randomly selected the perspective nuclear localization of Nrf2 and DAPI cells were under the microscope.

\section{Statistical analysis}

Results are expressed as means \pm S.E.M. Statistical differences were assessed with the unpaired 2-tailed Student's $t$-test for two experimental groups and one-way ANOVA for multiple groups with SPSS software. Bonferroni's post hoc testing was employed after ANOVA for testing for significant differences between groups. A two-tailed $P$ value of less than 0.05 was considered statistically significant. Statistical analyses were done using GraphPad Prism (GraphPad Software).

\section{Results}

\section{Baicalin attenuates hyperglycemia-induced endothelial dysfunction both in vivo and in vitro}

To demonstrate the protective effect of Baicalin against hyperglycemia-induced endothelial impairment in vivo, immunofluorescence staining for CD31 (an endothelial cell marker) revealed the presence of de-endothelialized region in aortal endothelium of diabetic mice induced by STZ $(100 \mathrm{mg} / \mathrm{kg}$ body weight, i.p.) compared with its corresponding control littermates. Systemic treatment of Baicalin $(50 \mathrm{mg} / \mathrm{kg} /$ day, i.p.) significantly attenuated hyperglycemia-induced de-endothelialization compared with vehicle-treated group in diabetic mice (Fig. 1A and B).

Endothelial cell apoptosis is a representative symptom of diabetic endothelial cell impairment and a leading cause of diabetic vascular disease (Kim et al. 2010). Thus, we analyzed the effect of intraperitoneal Baicalin injection on hyperglycemia-induced vascular apoptosis in the diabetic aortal endothelial cell. In diabetic mice, we found obvious accumulation of apoptosis puncta colocalized with aortal vascular endothelium (labeled with CD31) as compared with its corresponding control littermates. However, Baicalin-treated diabetic mice had significantly fewer apoptosis puncta when compared with vehicle-treated group (Fig. 1C and D).

To further determine the endothelial protective function of Baicalin, an ex vivo model, the aortic ring assay (Brill et al. 2004) was utilized. The aortic rings from C57BL/6 mice were cultured in different mediums containing NG $(5.5 \mathrm{mM})$ and $\mathrm{HG}(33 \mathrm{mM})$ alone or with Baicalin. In NG medium, we observed a well-structured microvessel network with clearly defined tubules and regular branching. In contrast, the rings cultured in HG medium showed a dramatically impaired sprouting function when compared with rings cultured in normal medium or in osmotic control group, while the sprouting function was well preserved by Baicalin administration (Fig. 1E and F).

In parallel, the tube-forming activity was also significantly impaired in HUVECs exposed to $H G$ as compared with HUVECs maintained in NG, but greatly enhanced by Baicalin (Fig. $1 \mathrm{G}$ and $\mathrm{H}$ ). Furthermore, hyperglycemia-induced high levels of apoptosis in HUVECs, as demonstrated by increase in the proportion of TUNEL-positive cells (Fig. 1I and J) and elevated protein levels of c-Caspase 3 and $\mathrm{Bax} / \mathrm{Bcl}-2$ ratio. However, hyperglycemia-induced apoptosis was significantly alleviated by Baicalin (Fig. 1K, L and M). Overall, a directly protective role of Baicalin against hyperglycemia-induced endothelial impairment was confirmed.

\section{Baicalin attenuates hyperglycemia-induced oxidative stress and inflammation via activating Nrf2-mediated exogenous antioxidant defenses both in vivo and in vitro}

It has been reported that endothelial dysfunction caused by hyperglycemia is mediated through several mechanisms including increased oxidative stress and pro-inflammatory responses (Xie et al. 2008). Therefore, we measured the effect of Baicalin on oxidative stress in 


\begin{tabular}{l|l|l|r|r|}
$\begin{array}{l}\text { Journal of } \\
\text { Endocrinology }\end{array}$ & G Chen, X Chen et al. & $\begin{array}{l}\text { Baicalin improve angiogenesis } \\
\text { in diabetes }\end{array}$ & $\mathbf{2 4 0 : 1}$ & $\mathbf{8 6}$ \\
\hline
\end{tabular}
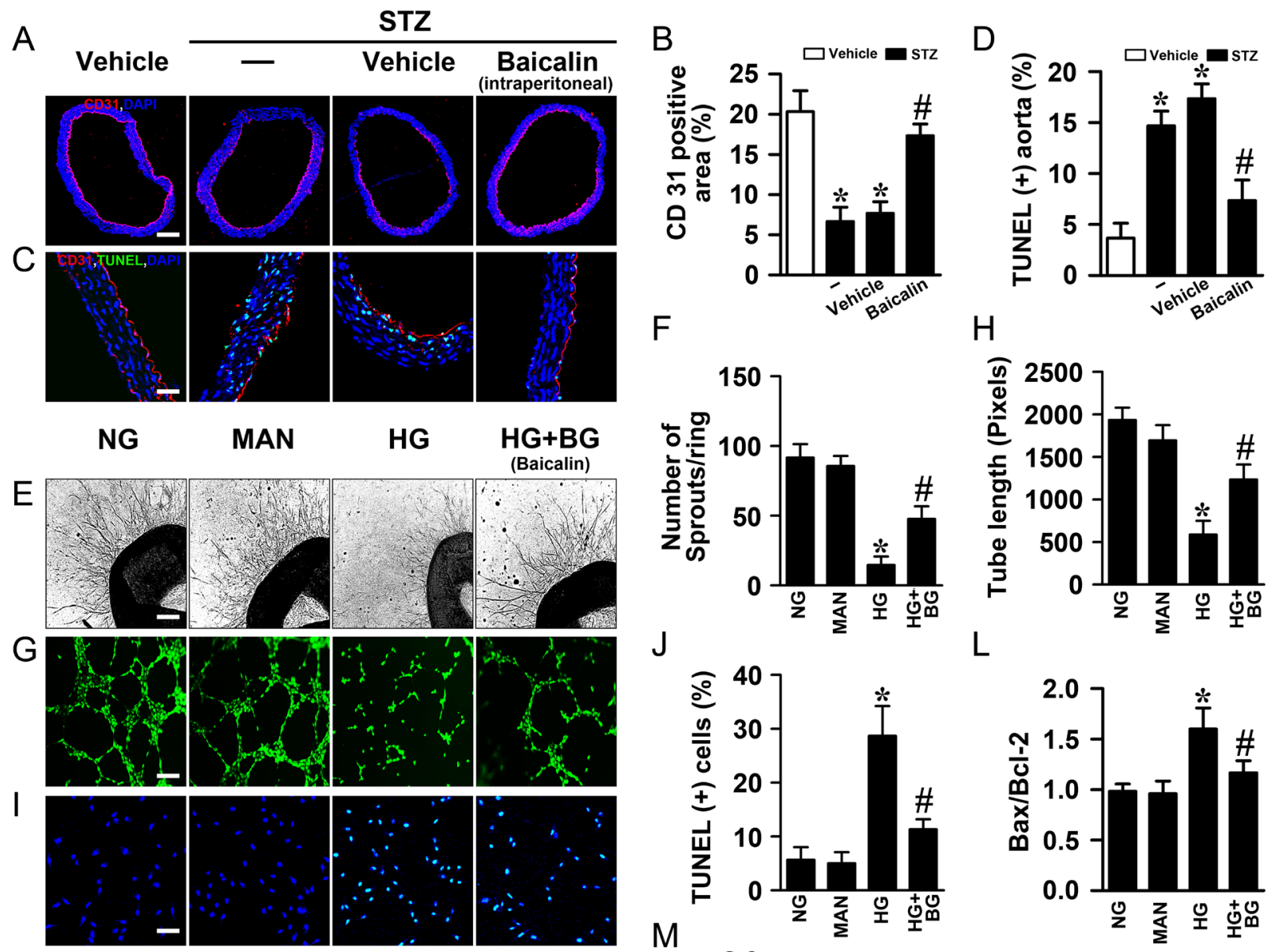

$\mathrm{F}$

$\mathrm{H}$
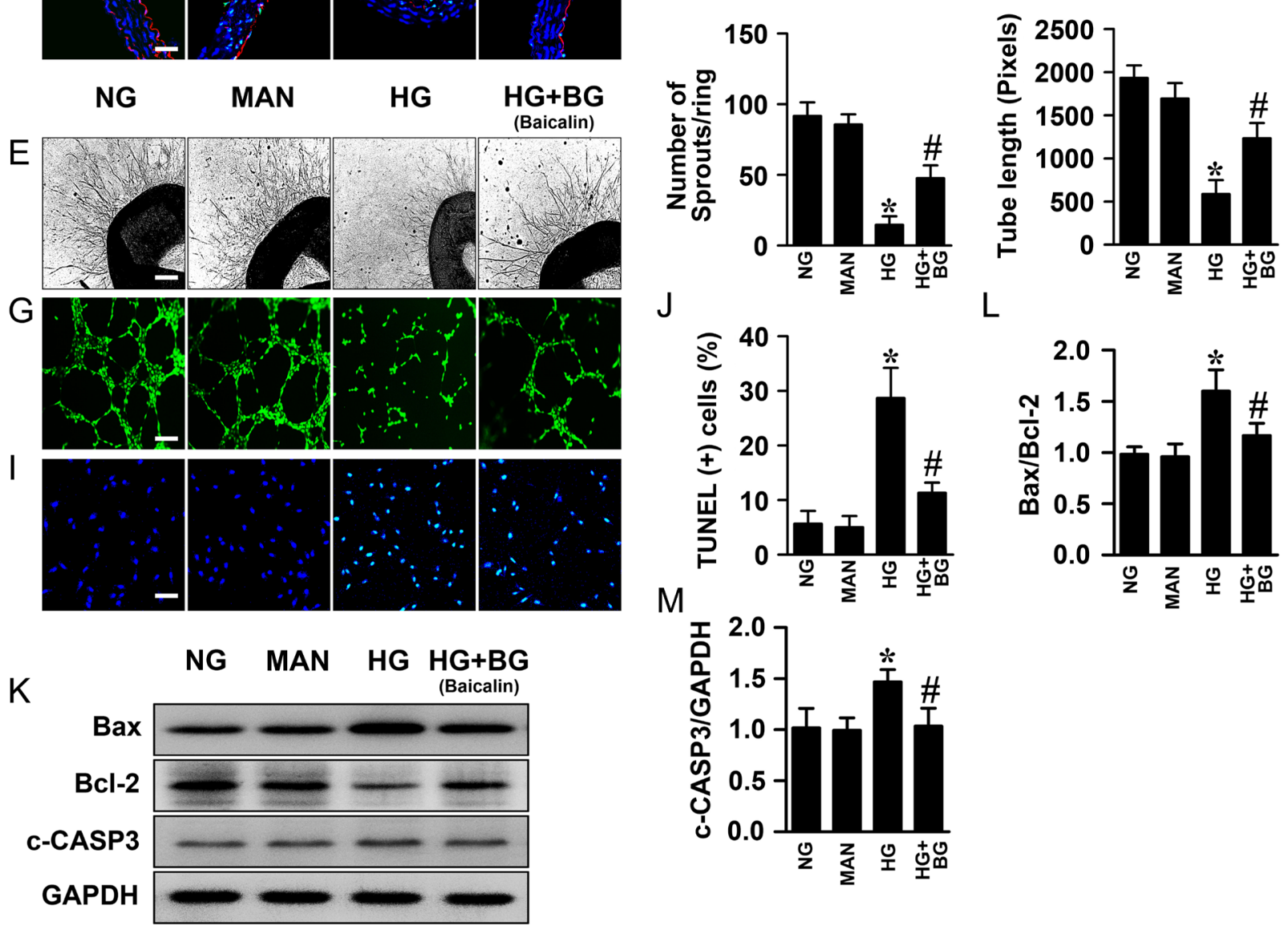

$\mathrm{M}$
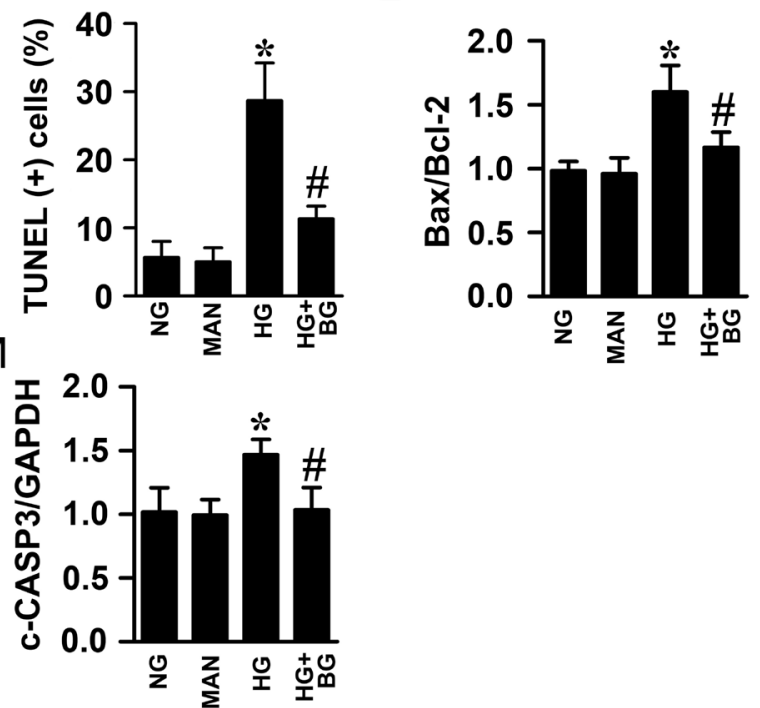

Figure 1

Baicalin attenuates hyperglycemia-induced endothelial dysfunction both in vivo and in vitro. (A) Representative immunofluorescence with CD31 from C57BL/6 mice received sodium citrate buffer $(\mathrm{pH} 4.5)$ injections, STZ-induced diabetic mice in the presence or absence of DMSO injections and Baicalintreated STZ-induced diabetic mice at aorta tissue sections. The red area represented endothelium and the nucleus was blue. Scale bars $=200 \mu \mathrm{m}$. (B) Quantification of the CD31-positive area in (A), values displayed are means \pm S.E.M. of eight independent experiments. ${ }^{*} P<0.05$ vs C57BL/6 mice; $\# P<0.05$ vs diabetic mice or vehicle-treated diabetic mice. (C) Representative confocal images of apoptosis in aortal vascular endothelium from C57BL/6 mice treated as indicated in (A). Scale bars $=40 \mu \mathrm{m}$. (D) The quantitative analysis of TUNEL+ cells in at least six separate fields, values displayed are means \pm S.E.M. of six independent experiments. ${ }^{*} P<0.05$ vs C57BL/ 6 mice; $\# P<0.05$ vs diabetic mice or vehicle treated diabetic mice. (E) Representative images of aortic rings from C57BL/6 mice cultured in different mediums containing NG $(5.5 \mathrm{mM})$, HG (33 mM) alone or with Baicalin ( $50 \mu \mathrm{M})$ for $72 \mathrm{~h}$, MAN ( $33 \mathrm{mM}$ : $5.5 \mathrm{mM}$ of glucose $+27.5 \mathrm{mM}$ of D-mannitol) was served as the osmotic control for the HG. Scale bars $=200 \mu \mathrm{m}$. (F) Quantification of the number of sprouts in (E), values displayed are means \pm S.E.M. of ten independent experiments. ${ }^{*} P<0.05$ vs NG or MAN; $\# P<0.05$ vs HG. (G) Capillary-like tube formation was assessed by Matrigel angiogenesis assay in HUVECs. HUVECs were cultured either in NG or HG medium in the presence or absence of Baicalin $(50 \mu \mathrm{M})$ for $72 \mathrm{~h}$, MAN was served as the osmotic control for the HG. Scale bars $=300 \mu \mathrm{m}$. (H) Quantification of the tube length in (G), images of tube morphology were taken in six random microscopic fields per sample and values displayed are means \pm S.E.M. of eight independent experiments. $* P<0.05$ vs NG or MAN; $\# P<0.05$ vs HG. (I) TUNEL assay of HUVECs treated as indicated in (G). The apoptotic cells were labeled with green, and nuclei were stained with DAPI (blue). Scale bars $=100 \mu \mathrm{m}$. () The quantitative analysis of TUNEL+ cells in at least six separate fields, values displayed are means \pm S.E.M. of six independent experiments. ${ }^{*} P<0.05$ vs NG or MAN; $\# P<0.05$ vs HG. (K) Cell lysates of HUVECs were used to detect Bax, BCl-2 as well as c-Caspase 3 protein levels by immunoblotting. ( $L$ and $M)$ The quantitative analysis of each immunoblots, the results were normalized to HUVECs exposed to NG, values displayed are means \pm S.E.M. of six independent experiments. ${ }^{*} P<0.05$ vs NG or MAN; $\# P<0.05$ vs HG. 
endothelial cells by DHE assay. Stimulation of HUVECs with HG caused a significant increase in production of superoxide, and this increase was alleviated by co-treatment with Baicalin (Fig. $2 \mathrm{~A}$ and B). Using 3-nitrotyrosine (3-NT) as an oxidative damage marker, coincidentally, Baicalin also reduced 3-NT levels that were elevated by HG exposure (Fig. 2E and F). To confirm the effect of Baicalin on hyperglycemia-induced superoxide production observed in vitro, the same strategy was performed on STZ-treated diabetic mice. We found that immunofluorescence staining for 3-NT was dramatically increased in aortal vascular endothelium from diabetic mice compared with vehicle-treated group. By contrast, Baicalin largely decreased the degree of vascular oxidative damage compared with vehicle-treated group in diabetic mice (Fig. 2C and D).

As Nrf2 plays a critical role in the cellular response to oxidative stress (Jyrkkänen et al. 2008). We speculate that Baicalin may activate Nrf2 and its downstream target genes to alleviate hyperglycemia-induced endothelial impairment. According to the results, there was no significant change in total Nrf2 protein level under any treatment condition in HUVECs (Fig. 2G and J). When treated with HG, however, the nuclear Nrf2 level was significantly declined but not observed by Baicalin co-treatment (Fig. 2G, H and I). In addition, the nuclear localization of Nrf2 was further proved by immunofluorescence in HUVECs (Fig. 2K). Overall, these results indicate that Baicalin promoted the nucleus enrichment of Nrf2 but not alter the total Nrf2 expression.

Importantly, we found that the mRNA levels for Nrf2 target genes HO1, NQO1, NQO2, SOD2 and CAT were significantly decreased by $\mathrm{HG}$ treatment, but especially alleviated by Baicalin (Fig. 3A, B, C, D, E and F). Hyperglycemia-induced oxidative damage is a critical intermediate for NF- $\mathrm{KB}$ activation, which plays a critical regulating role in the release of pro-inflammatory cytokines such as IL6, IL8 and TNFa (Rask-Madsen \& King 2013). Accordingly, we testified the mRNA expression of NF- $\mathrm{KB}$ target genes and found they were significantly increased in HUVECs exposed to HG as compared with HUVECs maintained in NG, but especially ameliorated by Baicalin or the pharmacological antioxidant molecules NAC (Fig. 3G, H, I, J and K). Coincidentally, there was no significant difference between Baicalin and NAC in the effect of anti-inflammation and reducing the proinflammatory cytokines such as IL6, IL8 and TNFa, which demonstrated that the downregulation of hyperglycemia elevated ROS and inflammation by Baicalin treatment is Nrf2 dependent. In conclusion, the changes of Nrf2 target gene accompany with the Nrf2 nuclear localization were induced by Baicalin (Figs 2G, H and I; 3A, B, C, D, E and $F)$, which demonstrated that Baicalin activates Nrf2 transcriptional function and attenuates hyperglycemiainduced oxidative stress and inflammation.

\section{Baicalin activates Nrf2 via Akt/GSK3B/Fyn pathway both in vivo and in vitro}

Baicalin is thought to attenuate hyperglycemia-induced embryonic cardiovascular malformation (Wang et al. 2018) and ketamine-induced neurotoxicity in the developing rats via Akt signaling (Zuo et al. 2016), and it also regulates antioxidant enzyme gene expression (Kim et al. 2012) by $\mathrm{Akt} / \mathrm{FoxO} 1$ signaling. In addition, the function of Nrf2 was reported to be regulated by the Akt/GSK3B/Fyn pathway in fibroblasts and PC12 cells by controlling Fyn-mediated export and degradation of nuclear Nrf2 (Li et al. 2014).

Consistently, we found that HG significantly increased the level of Fyn in the nucleus and decreased the phosphorylation of Akt and GSK3B in HUVECs. However, Baicalin attenuated hyperglycemia-induced decreases in Akt and GSK3B phosphorylation and nuclear accumulation of Fyn (Fig. 4A and B). Moreover, the effects of Baicalin on Nrf2 nuclear import, inducible expression of a battery of intrinsic antioxidant genes (Fig. 4C and D), decreased expression of NF-кB target genes at mRNA levels (Fig. 4E and F) and Fyn cytosolic translocation (Fig. 4A and B) were significantly prevented by the specific pharmacological inhibitor of PI3K, LY294002, through the inhibition of Akt and GSK3B phosphorylation. Subsequently, the protective function of Baicalin, including tube formation (Fig. 5C and D), preserve cell survival (Fig. 5E and F), anti-oxidative effect (Fig. 5G and $\mathrm{H}$ ) in HUVECs, lone with the vascular sprouting functions were abolished (Fig. 5A and B). Additionally, Baicalinmediated preservation of Akt/GSK3B/Fyn pathway was further confirmed in HAOECs (Fig. 6).

To demonstrate whether the protective effect of Baicalin against hyperglycemia-induced endothelial impairment via Akt/GSK3B/Fyn pathway in diabetic mice, LY294002 (5 mg/kg/day, i.p.) was administrated. As expected, inhibition of PI3K/Akt pathway by LY294002 largely counteracted Baicalin-modulated endothelial protective effect, which exhibited dramatically increasing vascular apoptosis (Fig. 5I and J), superoxide production (Fig. 5K and L) in aortal vascular endothelium cells from diabetic mice. These results suggest that Baicalin ameliorate endothelial function via regulating Akt/GSK3B/Fyn pathway to activate Nrf2 transcription. 
A

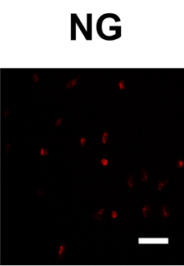

MAN

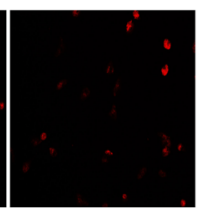

C

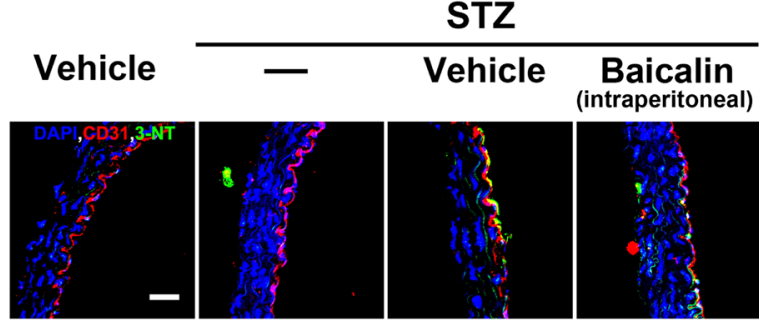

$G$
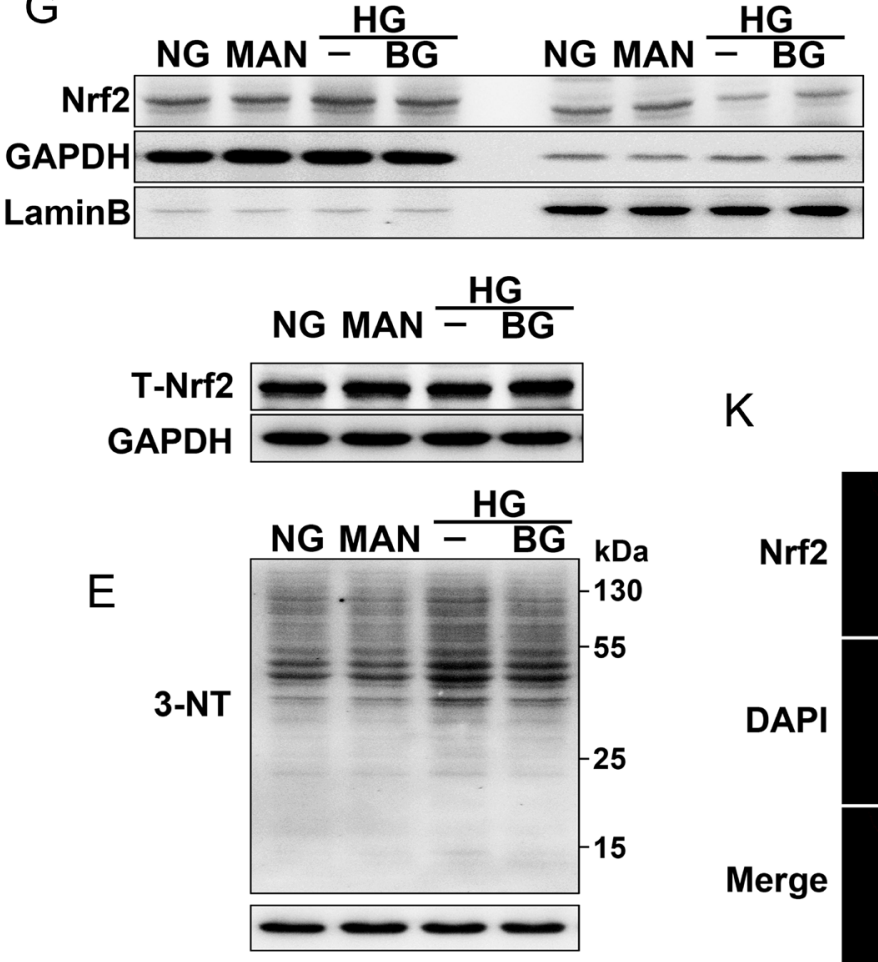

HG

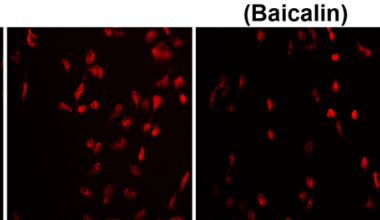

STZ (intraperitoneal)

F
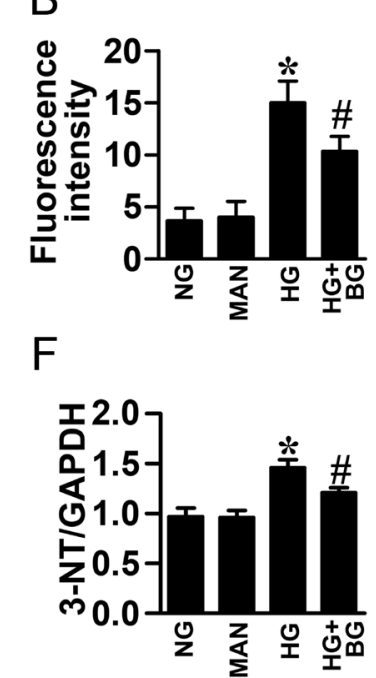

D
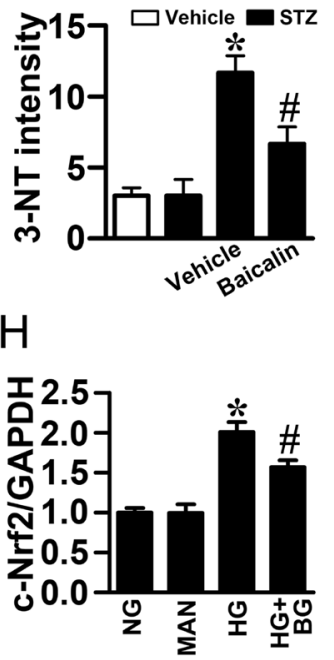

J

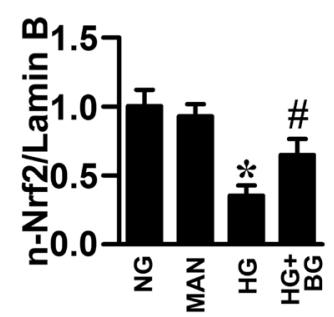

K

NG

MAN

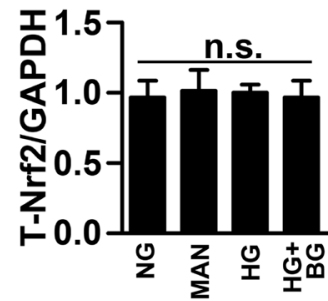

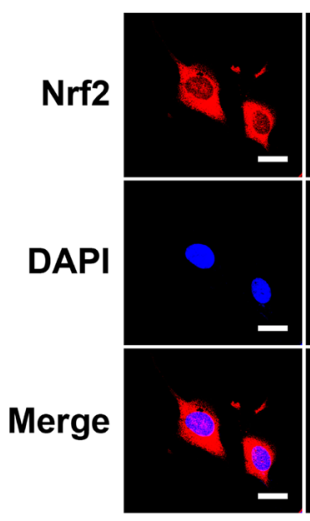
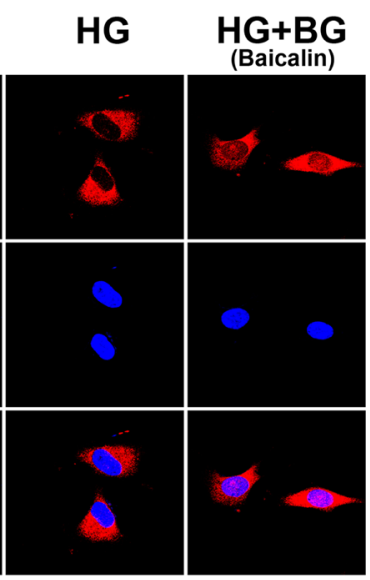

Figure 2

Baicalin attenuates hyperglycemia-induced oxidative stress and inflammation in HUVECs via activating Nrf2-mediated exogenous antioxidant defenses both in vivo and in vitro. (A) Fluorescent images and quantitation of superoxide levels in HUVECs cultured either in NG or HG medium in the presence or absence of Baicalin $(50 \mu \mathrm{M})$ for $72 \mathrm{~h}$, MAN was served as the osmotic control for the HG. Superoxide was determined with the fluorescent indicator DHE, and the fluorescent intensity of DHE was observed with a computer-assisted microscope (EVOS, Thermo Fisher Scientific) Scale bars $=100 \mu \mathrm{m}$. (B) The quantitative analysis of fluorescent intensity in at least six separate fields, values displayed are means \pm S.E.M. of six independent experiments. * $P<0.05$ vs NG or MAN; $\# P<0.05$ vs HG. (C) Representative confocal images of oxidative damage marker 3-NT in aortal vascular endothelium from C57BL/ 6 mice, diabetic mice, and intraperitoneal Baicalin (50 mg/kg/day) treated diabetic mice. The red area represented endothelium, the green area represents 3-NT positive staining and the nucleus was blue. Scale bars $=40 \mu \mathrm{m}$. (D) Quantification of the number of 3-NT staining in (C), values displayed are means \pm S.E.M. of eight independent experiments. ${ }^{*} P<0.05$ vs C57BL/ 6 mice; $\# P<0.05$ vs diabetic mice or vehicle treated diabetic mice. (E) Levels of the oxidative damage marker 3-NT in HUVECs treated with or without HG (33 mM) in the presence or absence of Baicalin ( $50 \mu \mathrm{M})$ for $72 \mathrm{~h}$ was detected by Western blot. MAN was served as the osmotic control for the HG. (F) The quantitative analysis of 3-NT protein immunoblots, the results were normalized to HUVECs exposed to NG, values displayed are means \pm s.E.M. of six independent experiments. * $P<0.05$ vS. NG or MAN; \#P<0.05 vs. HG. (G) Cytosolic (c-Nrf2) and nuclear (n-Nrf2) protein levels of Nrf2 and total (T-Nrf2) expression of Nrf2 were detected by Western blot, Which assay of HUVECs treated as indicated in (E). ( $\mathrm{H}, \mathrm{I}$ and J) The quantitative analysis of each immunoblots, the results were normalized to HUVECs exposed to NG, values displayed are means \pm S.E.M. of six independent experiments. ${ }^{*} P<0.05$ vs NG or MAN; ${ }^{*} P<0.05$ vs HG. (K) Nrf2 nuclear translocation was determined in fixed cells by immunofluorescent staining in HUVECs. The red area represented Nrf2 and the nucleus was blue. Scale bars $=20 \mu \mathrm{m}$. 

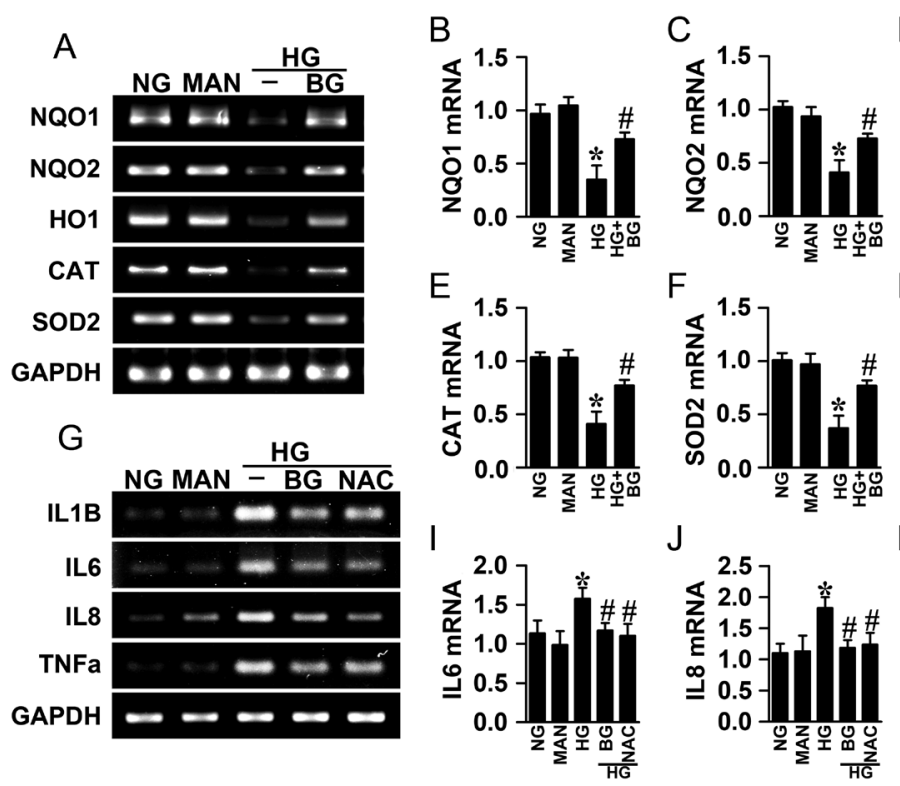

Knockdown or inhibitor of Nrf2 impairs the protective effects of Baicalin

To confirm the pivotal role of Nrf2 in the endothelial protective effect of Baicalin, Nrf2 gene expression was efficiently performed knockdown with adenoviral containing Nrf2 shRNA (Fig. 7A and B). In HUVECs, Nrf2shRNA eliminated the protective effect of Baicalin on nuclear accumulation of Nrf2 (Fig. 7C) and decreased the expression of target antioxidant genes (Fig. 7G, H, I, J, K and $\mathrm{L}$ ), which result in higher inflammation (Fig. 7M, N, $\mathrm{O}, \mathrm{P}$ and $\mathrm{Q}$ ), superoxide production (Figs 7E and $\mathrm{F} ; 8 \mathrm{E}$ and $\mathrm{F}$ ), apoptosis (Fig. 8C and D) and the damage of tube formation (Fig. 8A and B) under HG treatment conditions.

In addition, ML385, a specific inhibitor of Nrf2, substantially blocked Baicalin-mediated Nrf2 activity both in vivo and ex vivo, which effectively eliminated the protective ability of Baicalin in aortal vascular, including Nrf2-mediated effect of anti-oxidative (Fig. 8I and J) and anti-apoptosis (Fig. 8K and L), alone with a decreased vascular sprouting function (Fig. $8 \mathrm{G}$ and $\mathrm{H}$ ).

Moreover, although Nrf2 knockdown exerts no effect on the phosphorylation of Akt and GSK3B, and the reduced nuclear accumulation of the Fyn in HUVECs expose to HG with Baicalin (Fig. 9A, B, C, D and E), it dramatically decreased the expression of Nrf2 downstream target genes at mRNA levels (Fig. 9F, G, H, I, J and K). These results indicate the Nrf2 effects on the downstream of Akt/ GSK3B/Fyn pathway.

In addition, the endothelial protective action of Baicalin against hyperglycemia impairment might be mainly attributed to its role in activating Nrf2 via Akt/GSK3B/Fyn pathway.
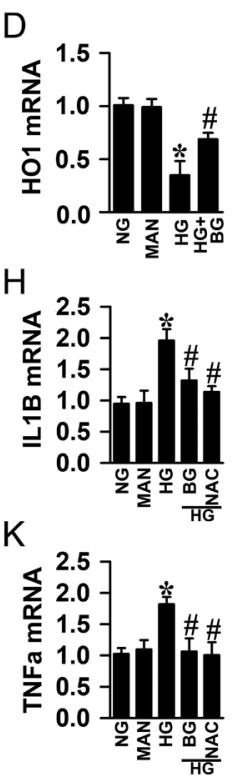

Figure 3

Baicalin attenuates hyperglycemia-induced oxidative stress and inflammation. HUVECs cultured either in NG or HG medium in the presence or absence of Baicalin for $72 \mathrm{~h}, \mathrm{MAN}$ was served as the osmotic control for the HG, NAC ( $2 \mathrm{mM}$ ) was pretreated for $2 \mathrm{~h}$ every day before Baicalin administration to evaluate the effect of Baicalin on the oxidative stress. (A, B, C, D, E and F) The mRNA expression and quantitation of Nrf2 downstream target genes were evaluated by sqRT-PCR. (G, H, I, J and K) The mRNA expression and quantitation of NF-кB downstream target genes were evaluated by sqRT-PCR. Two independent experiments were performed. Data shown in graphs represent the mean \pm S.D. $\star P<0.05$ vs NG or MAN; $\# P<0.05$ vs HG.

\section{Discussion}

Diabetes mellitus is a chronic metabolic disease with high blood glucose level and closely related to endothelial dysfunction, an important factor in the pathogenesis of vascular changes (Grundy et al. 2002, Erusalimsky 2009, Cho et al. 2018), accompanied by the production of ROS, which plays a causative role in the development and progression of virtually all diabetic vascular complications (Guzik et al. 2002, D’Autreaux \& Toledano 2007, Pitocco et al. 2013). Baicalin is a flavonol glycoside in Scutellaria baicalensis Georgi and has been extensively used as traditional medicine in many East Asian countries to reduce inflammation (Krakauer et al. 2001) and scavenging ROS (Lin et al. 2005, Waisundara et al. 2011). Earlier studies have shown that Baicalin possess wide-ranging biological and pharmacological functions, including anticancer, anti-pruritic effects (Li et al. 2011) and alleviate hyperglycemia (Li et al. 2011) as well as insulin resistance (Zou et al. 2005, Fang et al. 2017). However, the protective mechanism of Baicalin function involved in diabetes mellitus-induced endothelial cells dysfunction by activating Nrf2-mediated exogenous antioxidant defenses remains largely unknown.

The present study provides two new lines of evidence implicating Baicalin-initiated pathways in diabetic endothelium (HUVECs and HAOECs) dysfunction. The first innovative finding is that the benefit of Baicalin requires activation of Nrf2 antioxidant signaling. The second novel finding is that the Akt/GSK3B/Fyn pathway mediates Baicalin-induced Nrf2 activation in diabetic HUVECs and HAOECs. 

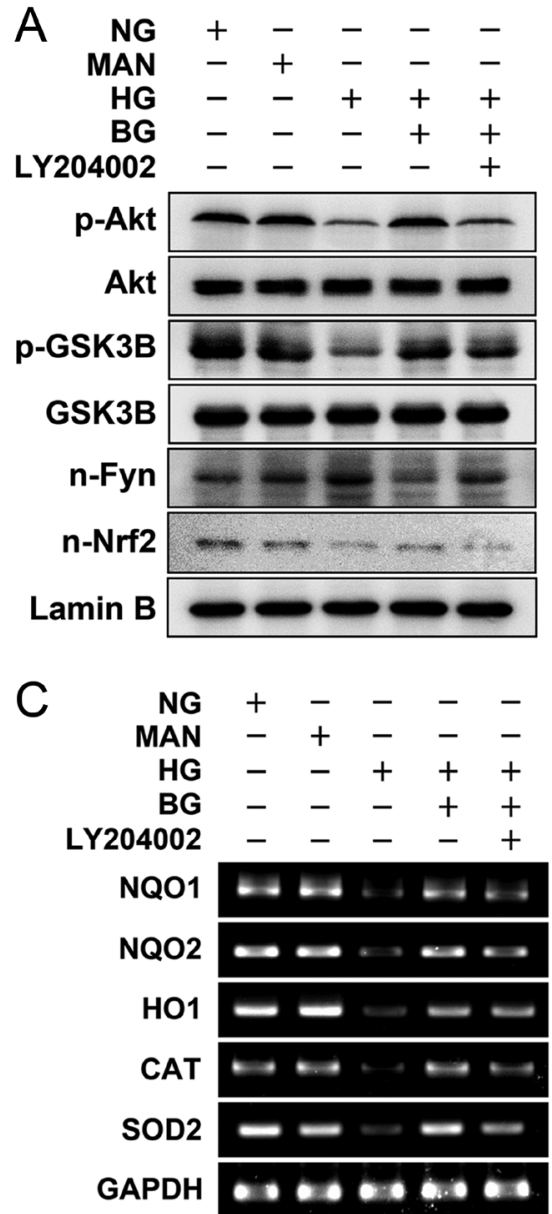
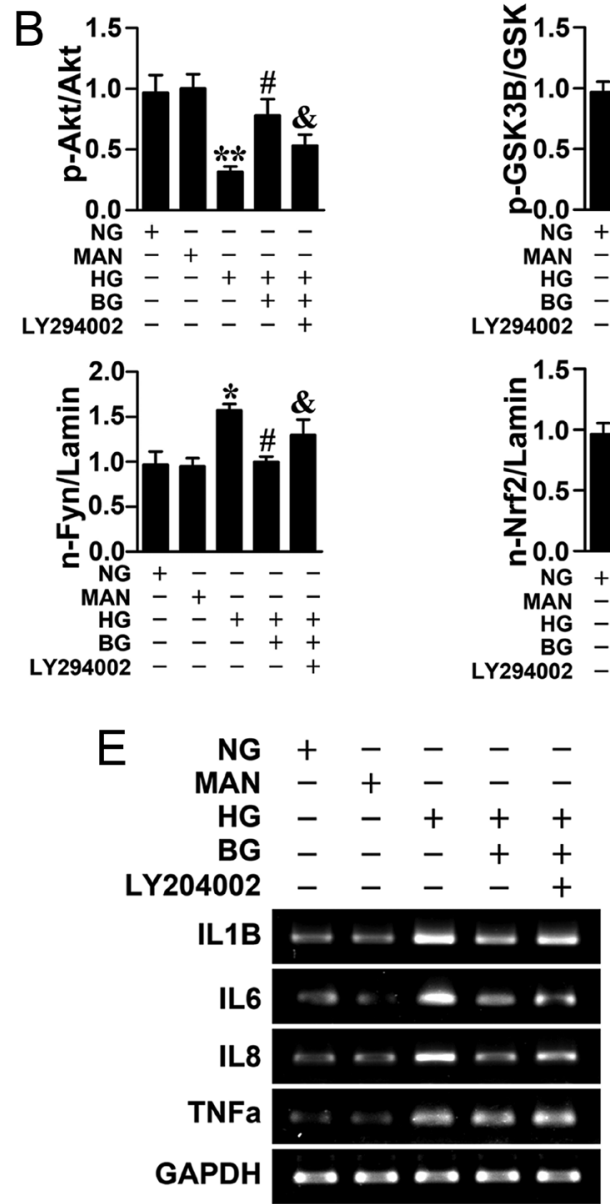

D
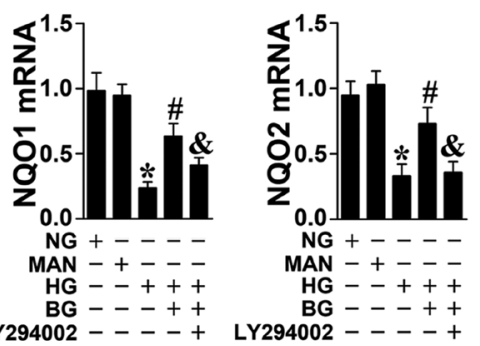

F
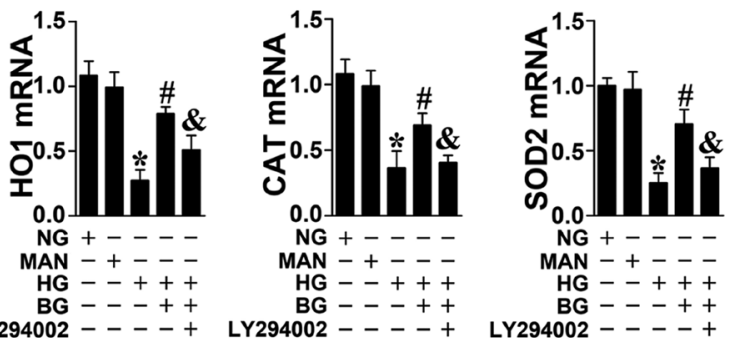

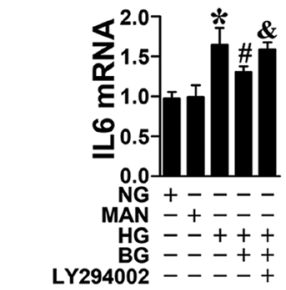

LY294002 - - - +
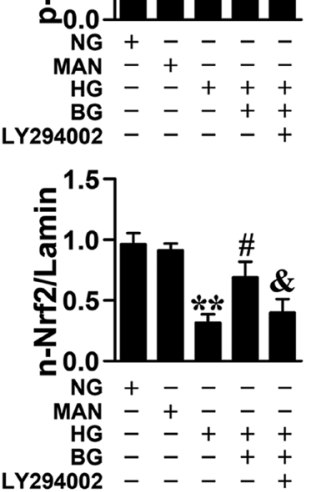

BG - - - +
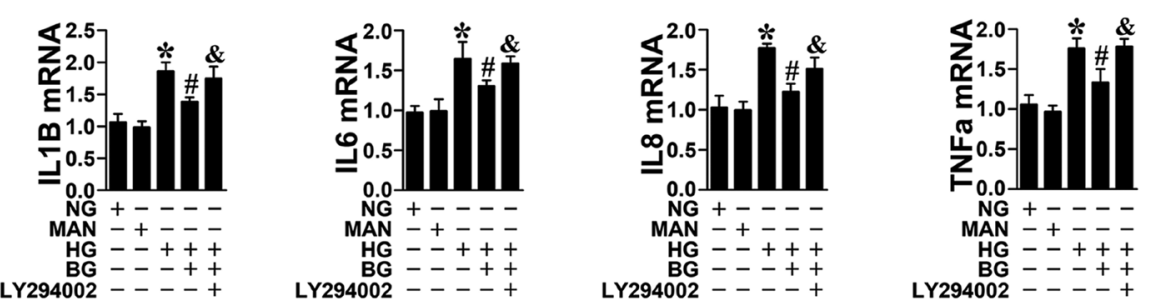

Figure 4

Baicalin activates Nrf2 via Akt/GSK3B/Fyn pathway in HUVECs. HUVECs were pretreated with or without LY294002 (10 $\mu$ M) for 30 min and then exposed to $\mathrm{HG}(33 \mathrm{mM})$ for $72 \mathrm{~h}$ in the presence or absence of Baicalin $(50 \mu \mathrm{M})$, MAN (33 mM: $5.5 \mathrm{mM}$ of glucose $+27.5 \mathrm{mM}$ of D-mannitol) was served as the osmotic control for the HG. (A) The phosphorylation of Akt and GSK3B, the nuclear translocation of Fyn (n-Fyn) and Nrf2 (n-Nrf2) were evaluated by Western blot. (B) The quantitative analysis of each immunoblots, the results were normalized to HUVECs exposed to NG, values displayed are means \pm S.E.M. of six independent experiments. $* P<0.05$ vs NG or MAN; $* * P<0.01$ vs NG or MAN; $\# P<0.05$ vs HG; \&P< 0.05 vs HG in the presence of Baicalin. (C and D) The mRNA expression and quantitation of Nrf2 downstream target genes were determined by sqRT-PCR. (E and F) The mRNA expression and quantitation of NF-кB downstream target genes were determined by sqRT-PCR. Two independent experiments were performed. Data shown in graphs represent the mean \pm S.D. ${ }^{*} P<0.05$ vs NG or MAN; $\# P<0.05$ vs HG; $\& P<0.05$ vs HG in the presence of Baicalin. 


\begin{tabular}{l|l|l|r|r|}
$\begin{array}{l}\text { Journal of } \\
\text { Endocrinology }\end{array}$ & G Chen, X Chen et al. & $\begin{array}{l}\text { Baicalin improve angiogenesis } \\
\text { in diabetes }\end{array}$ & $\mathbf{2 4 0 : 1}$ & $\mathbf{9 1}$ \\
\hline
\end{tabular}

A
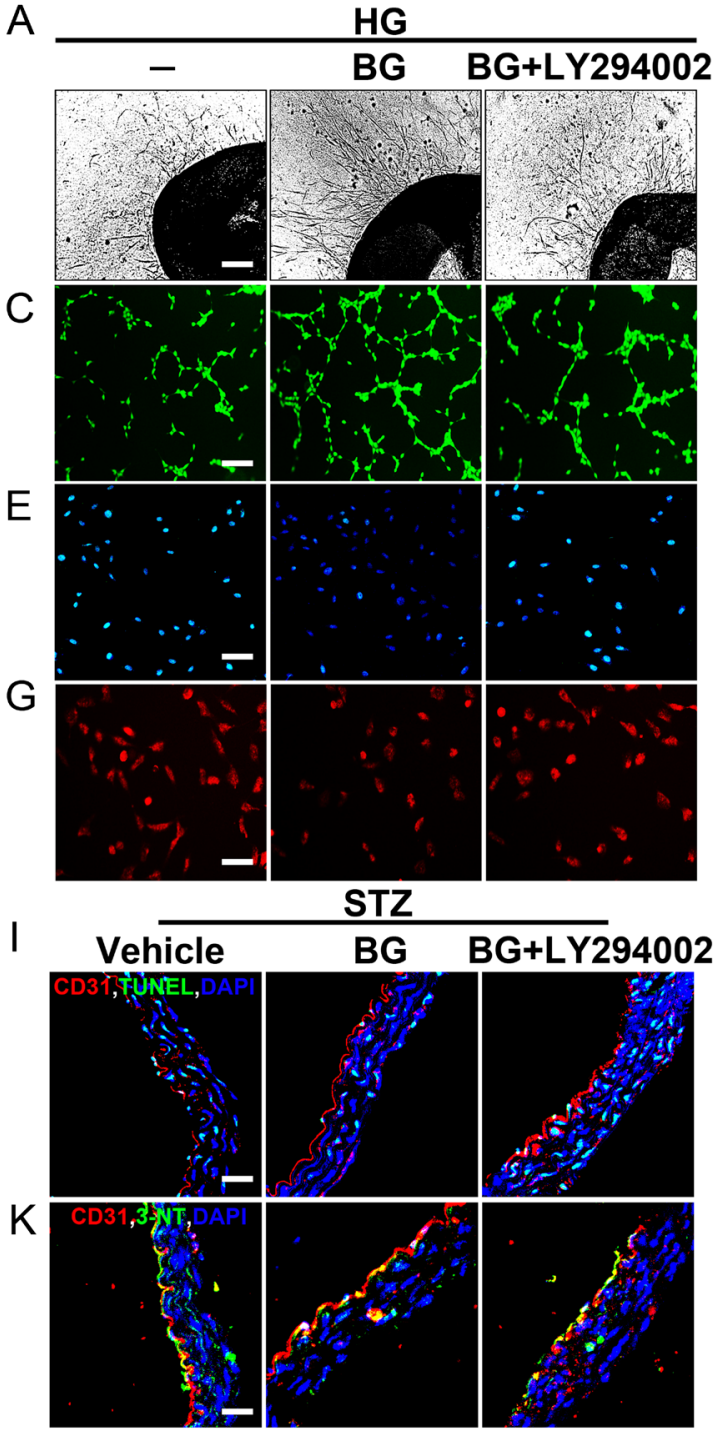

$B$

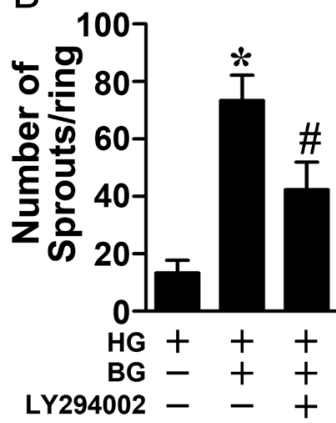

$\mathrm{F}$

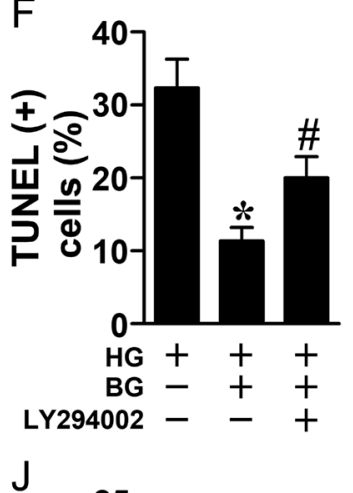

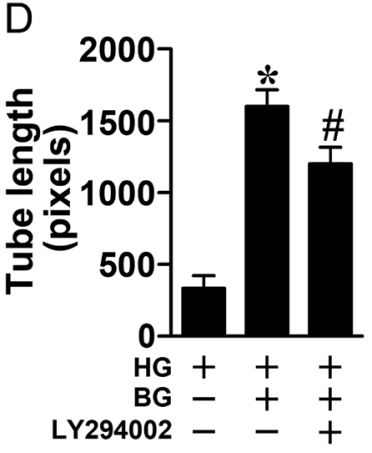

$\mathrm{H}$

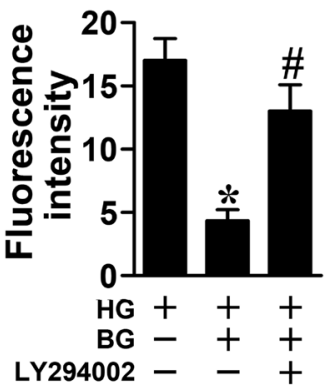

$\mathrm{L}$

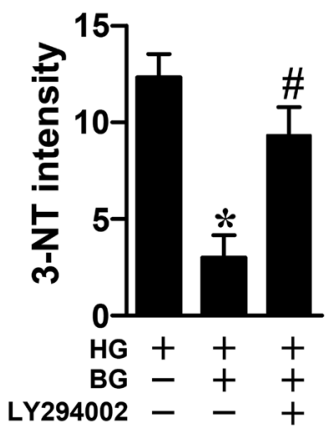

Figure 5

Baicalin activates Nrf2 via Akt/GSK3B/Fyn pathway both in vivo and in vitro. (A) Representative images of aortic rings from C57BL/6 mice were pretreated with or without inhibitor LY294002 $(10 \mu \mathrm{M})$ for $30 \mathrm{~min}$ and then exposed to HG $(33 \mathrm{mM})$ in the presence or absence of Baicalin $(50 \mu \mathrm{M})$. Scale bars $=200 \mu \mathrm{m}$. (B) Quantification of the number of sprouts in (A), values displayed are means \pm S.E.M. of ten independent experiments. $* P<0.05$ vs $H G$; $\# P<0.05$ vs HG in the presence of Baicalin. (C) Capillary-like tube formation was assessed by Matrigel angiogenesis assay in HUVECs. HUVECs were cultured with or without LY294002 $(10 \mu \mathrm{M})$ and then exposed to HG $(33 \mathrm{mM})$ medium in the presence or absence of Baicalin $(50 \mu \mathrm{M})$ for $72 \mathrm{~h}$. Scale bars $=300 \mu \mathrm{m}$. (D) Quantification of the tube length in (C), images of tube morphology were taken in six random microscopic fields per sample and values displayed are means \pm S.E.M. of eight independent experiments. $* P<0.05$ vs HG; $\# P<0.05$ vs HG in the presence of Baicalin. (E) TUNEL assay of HUVECs treated as indicated in (C). The apoptotic cells were labeled with green, and nuclei were stained with DAPI (blue). Scale bars $=100 \mu \mathrm{m}$. (F) The quantitative analysis of TUNEL+ cells in at least six separate fields, values displayed are means \pm S.E.M. of six independent experiments. $* P<0.05$ vs HG; $\# P<0.05$ vs HG in the presence of Baicalin. (G) Superoxide product test assay of HUVECs treated as indicated in (C), Superoxide was determined with the fluorescent indicator DHE, and the fluorescent intensity of DHE was observed with a computer-assisted microscope. Scale bars $=100 \mu \mathrm{m}$. (H) The quantitative analysis of fluorescent intensity in at least six separate fields, values displayed are means \pm S.E.M. of six independent experiments. ${ }^{*} P<0.05$ vs $\mathrm{HG}$; $\# P<0.05$ vs $\mathrm{HG}$ in the presence of Baicalin. (I) Representative confocal images of apoptosis in aortal vascular endothelium from vehicle treated diabetic mice, and intraperitoneal Baicalin ( $50 \mathrm{mg} / \mathrm{kg} /$ day) treated diabetic mice in the presence or absence of LY294002 (5 mg/kg/day). Scale bars $=40 \mu \mathrm{m}$. (J) The quantitative analysis of TUNEL+ cells in at least six separate fields, values displayed are means \pm s.E.M. of six independent experiments. $* P<0.05$ vs diabetic mice; $\# P<0.05$ vs diabetic mice in the presence of Baicalin. (K) Representative confocal images of oxidative damage marker 3-NT in aortal vascular endothelium from diabetic mice treated as indicated in (I). The red area represented endothelium, the green area represent 3-NT positive staining and the nucleus was blue. Scale bars $=40 \mu \mathrm{m}$. (L) Quantification of the number of 3-NT staining in (K), values displayed are means \pm S.E.M. of eight independent experiments. ${ }^{*} P<0.05$ vs diabetic mice; $\# P<0.05$ vs diabetic mice in the presence of Baicalin. 


\begin{tabular}{l|l|l|l|l|}
$\begin{array}{l}\text { Journal of } \\
\text { Endocrinology }\end{array}$ & G Chen, X Chen et al. & $\begin{array}{l}\text { Baicalin improve angiogenesis } \\
\text { in diabetes }\end{array}$ & $\mathbf{2 4 0 : 1}$ & $\mathbf{9 2}$ \\
\hline
\end{tabular}

A

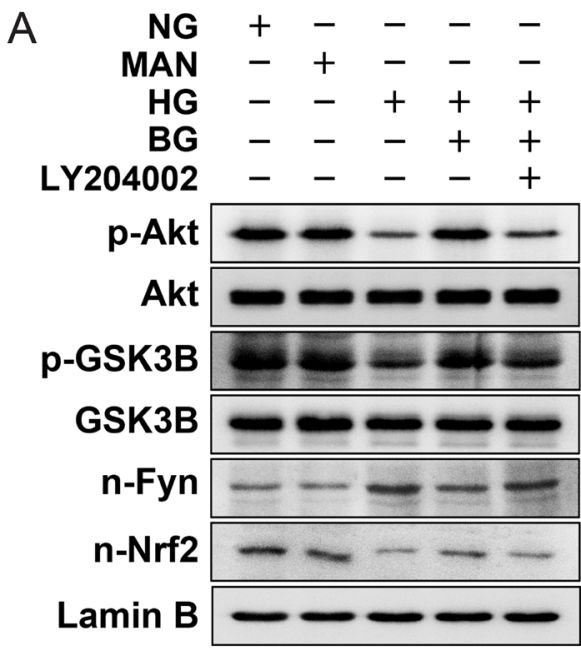

C

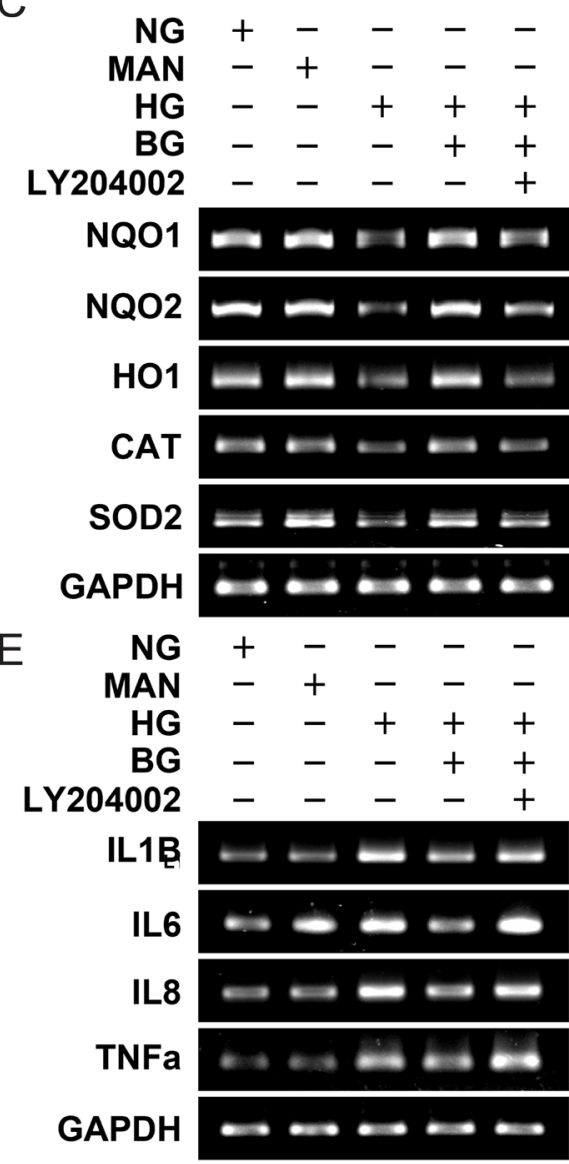

B
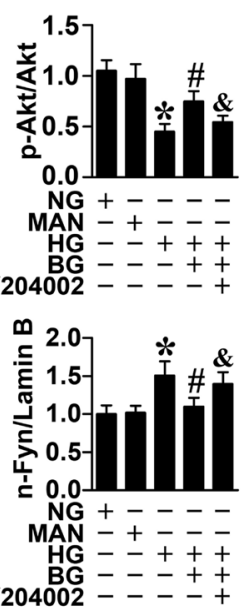

LY204002 $=-2++$

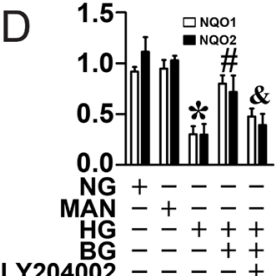

LY204002

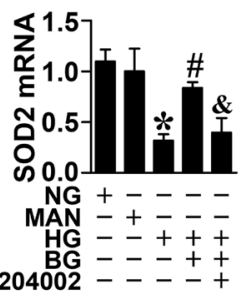

$204002---++$

G

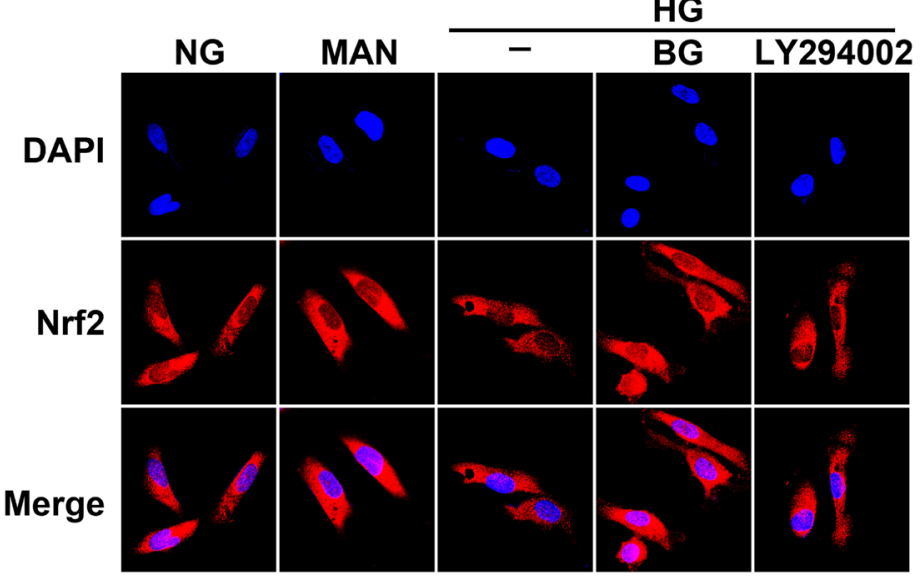

Figure 6

Baicalin-mediated preservation of Akt/GSK3B/Fyn pathway was further confirmed in HAOECs. HAOECs were pretreated with or without LY294002 for $30 \mathrm{~min}$ and then exposed to $\mathrm{HG}$ for $72 \mathrm{~h}$ in the presence or absence of Baicalin, MAN was served as the osmotic control for the HG. (A) The phosphorylation of Akt and GSK3B, the nuclear translocation of n-Fyn and n-Nrf2 were evaluated by Western blot. (B) The quantitative analysis of each immunoblots, the results were normalized to HAOECs exposed to NG, values displayed are means \pm s.E.M. of six independent experiments. $* P<0.05$ vs NG or MAN; $\# P<0.05$ vs HG; \&P $<0.05$ vs HG in the presence of Baicalin. (C and D) The expression and quantitation of Nrf2 downstream target genes were evaluated by sqRT-PCR. The results were normalized to HAOECs exposed to NG, values displayed are means \pm S.E.M. of six independent experiments. $* P<0.05$ vs NG or MAN; $\# P<0.05$ vs HG; $\& P<0.05$ vs HG in the presence of Baicalin. ( $E$ and $F$ ) The mRNA expression and quantitation of NF-kB downstream target genes were determined by SQRT-PCR. The results were normalized to HAOECs exposed to NG, values displayed are means \pm S.E.M. of six independent experiments. $* P<0.05$ vs NG or MAN; $\# P<0.05$ vs HG; $\& P<0.05$ vs HG in the presence of Baicalin. (G) Nrf2 nuclear translocation was determined in fixed cells by immunofluorescent staining in HAOECs. The red area represented Nrf2 and the nucleus was blue. Scale bars $=20 \mu \mathrm{m}$. 


\begin{tabular}{l|l|l|l|l|}
$\begin{array}{l}\text { Journal of } \\
\text { Endocrinology }\end{array}$ & G Chen, X Chen et al. & $\begin{array}{l}\text { Baicalin improve angiogenesis } \\
\text { in diabetes }\end{array}$ & $\mathbf{2 4 0 : 1}$ & $\mathbf{9 3}$ \\
\hline
\end{tabular}

A

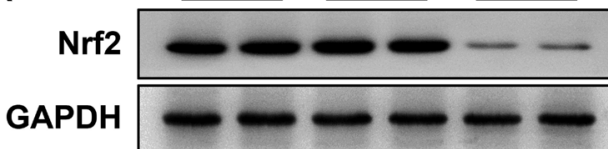

C

\begin{tabular}{|c|c|c|c|c|}
\hline MAN & + & - & - & - \\
\hline HG & - & + & + & + \\
\hline BG & - & - & + & + \\
\hline Scramble & + & + & + & \\
\hline sh-Nrf2 & - & - & - & + \\
\hline n-Nrf2 & 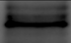 & $=$ & 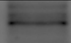 & \\
\hline Lamin B & $=$ & & & \\
\hline
\end{tabular}

G

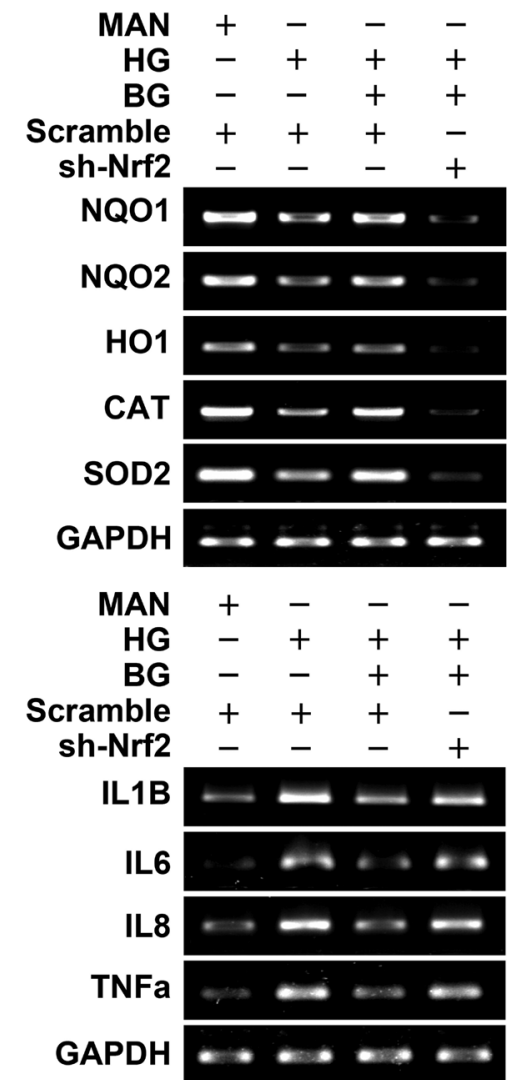

$$
\text { 竞 }
$$

D $\infty_{1}$
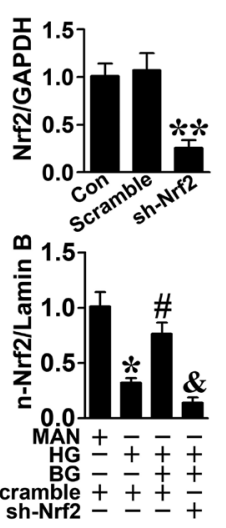

$\begin{array}{rrrrr}\text { E MAN } & + & - & - & - \\ \text { HG } & - & + & + & + \\ \text { BG } & - & - & + & + \\ \text { Scramble } & + & + & + & - \\ \text { sh-Nrf2 } & - & - & - & +\end{array}$

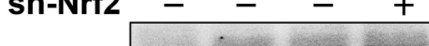

kDa

$-130$

$F$

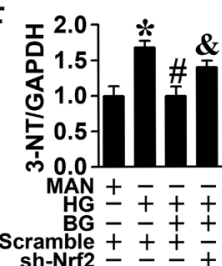

Scramble $+\overline{+}+ \pm$

$\mathrm{H}$

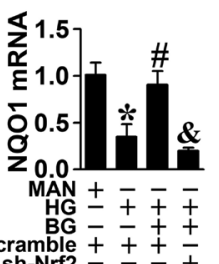

Scramble $\pm+ \pm \pm+\frac{+}{+}$

$\mathrm{K}$

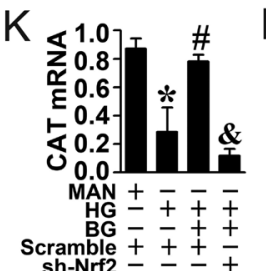

sh-Nrf2 $\pm \underline{+} \pm$

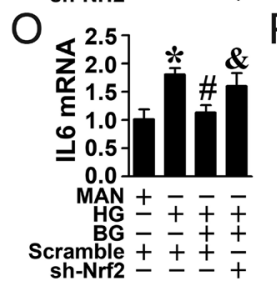

$-55$

3-NT
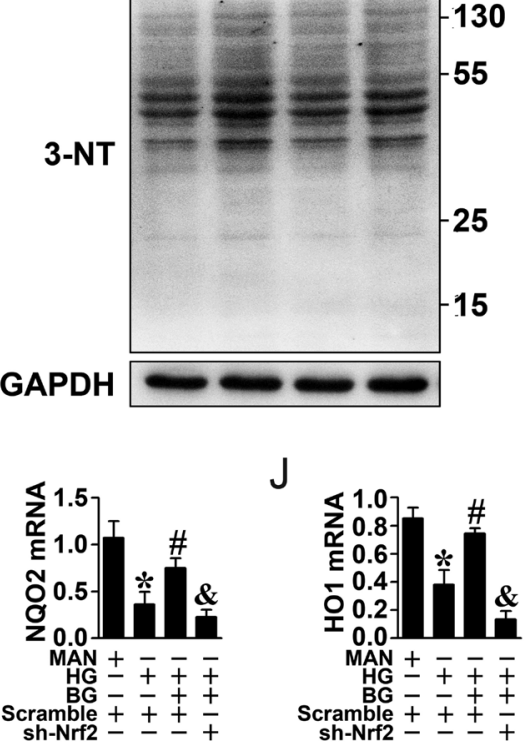

Sh-Nrf2 - \pm+
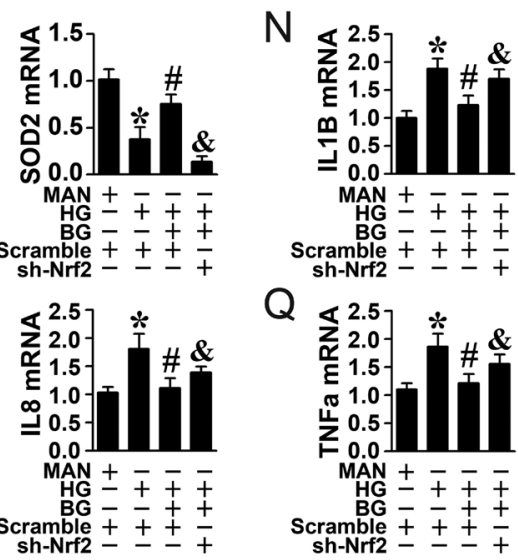

Figure 7

Knockdown of Nrf2 impairs the protective effects of Baicalin in HUVECs. (A) Protein expression of Nrf2 in HUVECs after transfecting with Nrf2 shRNA adenoviral vectors for $72 \mathrm{~h}$ assayed by Western blot to verify the efficacy of Nrf2 shRNA. (B) The quantitative analysis of Nrf2 immunoblots, the results were normalized to HUVECs transfected Ad-scramble, values displayed are means \pm S.E.M. of six independent experiments. ${ }^{*} P P<0.01$ vs HUVECS transfected Ad-scramble. (C) HUVECs were transduced with adenoviruses harboring sh-Nrf2 (Ad-sh-Nrf2) and a scrambled sequence (Ad-scramble) respectively. After transduction, HUVECs were cultured either in MAN or HG medium in the presence or absence of Baicalin for $72 \mathrm{~h}$. Protein expression of nuclear accumulation of Nrf2 in HUVECs after transfecting with adenoviruses assayed by Western blot. (D) The quantitative analysis of nuclear accumulation of Nrf2 immunoblots, the results were normalized to HUVECs transfected Ad-scramble expose to MAN. (E) Levels of the oxidative damage marker 3-NT in HUVECs was detected by Western blot. Which assay of HUVECs treated as indicated in (C). (F) The quantitative analysis of 3-NT protein immunoblots, the results were normalized to HUVECs transfected Ad-scramble. (G, H, I, J, K and L) The mRNA expression and quantitation of Nrf2 downstream target genes were determined by sqRT-PCR. Which assay of HUVECs treated as indicated in (C), the results were normalized to HUVECS transfected Ad-scramble. ( $\mathrm{M}, \mathrm{N}, \mathrm{O}, \mathrm{P}$ and Q) The mRNA expression and quantitation of NF-kB downstream target genes were determined by sqRT-PCR. Which assay of HUVECs treated as indicated in (C), the results were normalized to HUVECs transfected Ad-scramble. Data shown in graphs (C, D, E, F, G, $\mathrm{H}, \mathrm{I}, \mathrm{J}, \mathrm{K}, \mathrm{L}, \mathrm{M}, \mathrm{N}, \mathrm{O}, \mathrm{P}$ and Q) represent the means \pm S.E.M. of six independent experiments. * $P<0.05$ vs HUVECs transfected Ad-scramble expose to MAN; $\# P<0.05$ vs HUVECS transfected Ad-scramble expose to HG; \& $P<0.05$ vs HUVECs transfected Ad-scramble expose to HG in the presence Baicalin. 


\begin{tabular}{l|l|l|r|r|}
$\begin{array}{l}\text { Journal of } \\
\text { Endocrinology }\end{array}$ & G Chen, X Chen et al. & $\begin{array}{l}\text { Baicalin improve angiogenesis } \\
\text { in diabetes }\end{array}$ & $\mathbf{2 4 0 : 1}$ & $\mathbf{9 4}$ \\
\hline
\end{tabular}

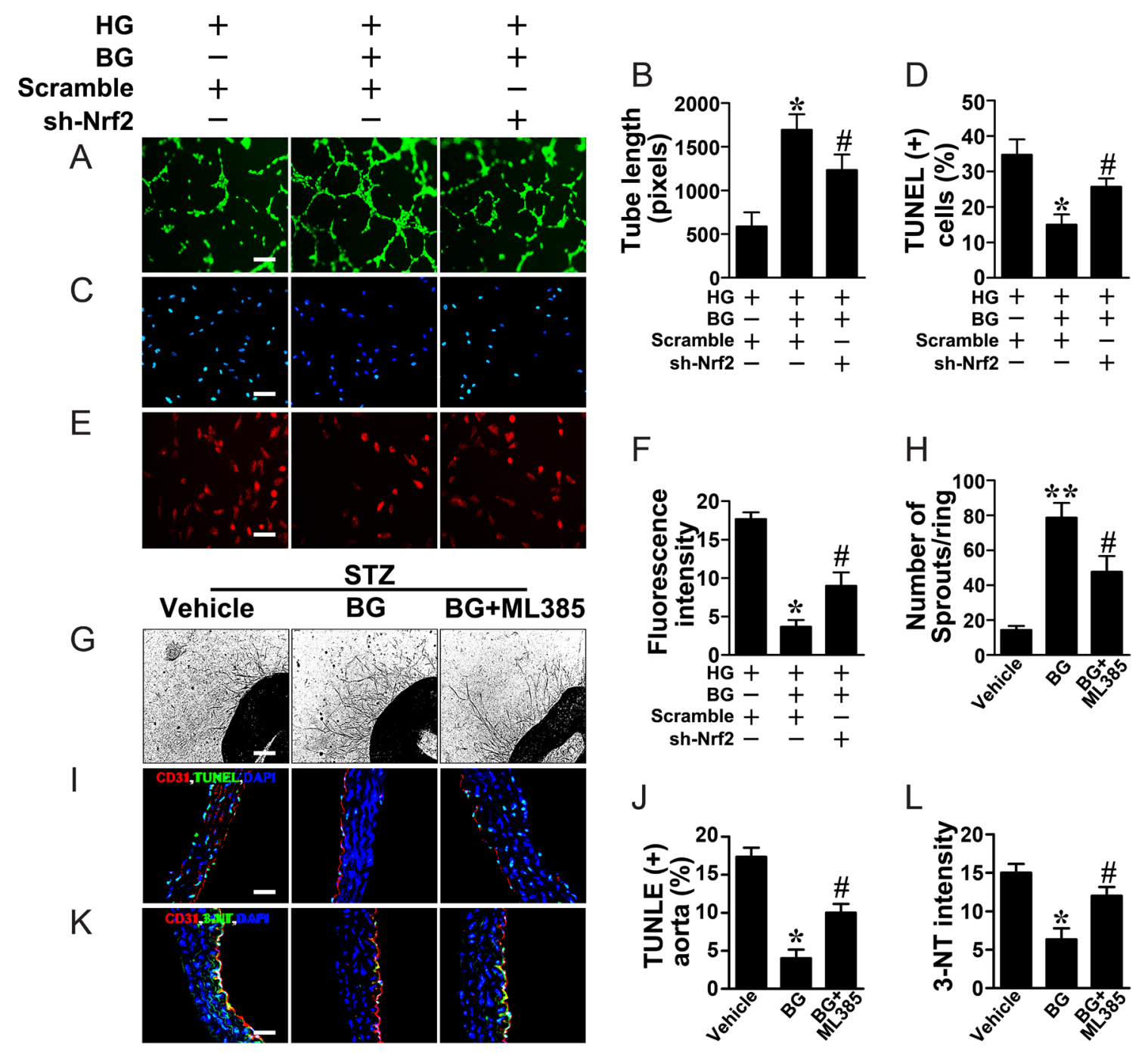

\section{Figure 8}

Knockdown or inhibitor of Nrf2 impairs the protective effects of Baicalin. (A) Capillary-like tube formation was assessed by Matrigel angiogenesis assay in HUVECS. HUVECs were transfected with or without adenoviral containing Nrf2 shRNA (Ad-sh-Nrf2) or scramble shRNA (Ad-scramble) and then exposed to $\mathrm{HG}$ medium in the presence or absence of Baicalin for $72 \mathrm{~h}$. Scale bars $=300 \mu \mathrm{m}$. (B) Quantification of the tube length in (A), images of tube morphology were taken in six random microscopic fields per sample and values displayed are means \pm S.E.M. of eight independent experiments. $* P<0.05$ vs Ad-scramble HUVECs; $\# P<0.05$ vs Ad-scramble HUVECs in the presence of Baicalin. (C) TUNEL assay of HUVECs treated as indicated in (A). The apoptotic cells were labeled with green, and nuclei were stained with DAPI (blue). Scale bars $=100 \mu \mathrm{m}$. (D) The quantitative analysis of TUNEL+ cells in at least six separate fields, values displayed are means \pm s.E.M. of six independent experiments. $* P<0.05$ vs Ad-scramble HUVECs HG; $\# P<0.05$ vs Ad-scramble HUVECs in the presence of Baicalin. (E) Superoxide product test assay of HUVECs treated as indicated in (A), Superoxide was determined with the fluorescent indicator DHE, and the fluorescent intensity of DHE was observed with a computer-assisted microscope. Scale bars $=100 \mu \mathrm{m}$. (F) The quantitative analysis of fluorescent intensity in at least six separate fields, values displayed are means \pm S.E.M. of six independent experiments. $* P<0.05$ vs Ad-scramble HUVECs HG; $\# P<0.05$ vs Ad-scramble HUVECs HG in the presence of Baicalin. (G) Representative images of aortic rings from diabetic mice were pretreated with or without Nrf2 inhibitor ML385 and Baicalin, then cultured with HG in the presence or absence of ML385 and Baicalin. Scale bars $=200 \mu \mathrm{m}$. (H) Quantification of the number of sprouts in $(G)$, values displayed are means \pm S.E.M. of ten independent experiments. $* P<0.05$ vs vehicle treated diabetic mice; $\# P<0.05$ vs diabetic mice in the presence of Baicalin. (I) Representative confocal images of apoptosis in aortal vascular endothelium from vehicle treated diabetic mice, and intraperitoneal Baicalin treated diabetic mice in the presence or absence of ML385. Scale bars $=40 \mu \mathrm{m}$. (J) The quantitative analysis of TUNEL + cells in at least six separate fields, values displayed are means \pm s.E.M. of six independent experiments. $* P<0.05$ vs. vehicle treated diabetic mice; $\# P<0.05$ vs diabetic mice in the presence of Baicalin. (K) Representative confocal images of oxidative damage marker 3-NT in aortal vascular endothelium from diabetic mice treated indicated in (I). The red area represented endothelium, the green area represent 3-NT positive staining and the nucleus was blue. Scale bars $=40 \mu \mathrm{m}$. (L) Quantification of the number of 3-NT staining in (K), values displayed are means \pm S.E.M. of eight independent experiments. ${ }^{*} P<0.05$ vs vehicle treated diabetic mice; $\# P<0.05$ vs diabetic mice in the presence of Baicalin. 


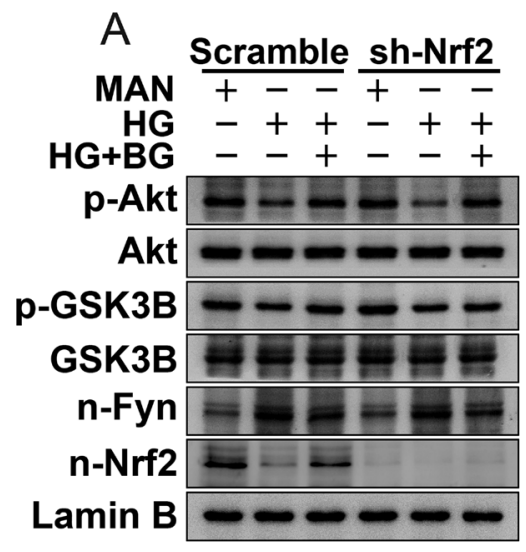

$\mathrm{F}$

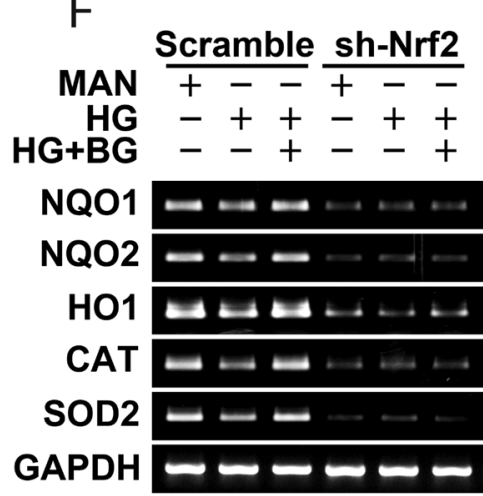

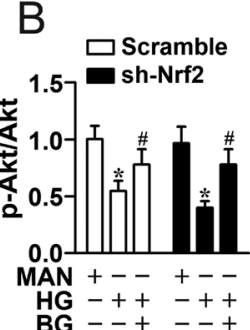

E

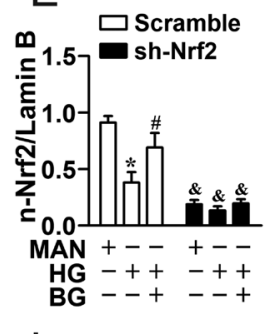

I

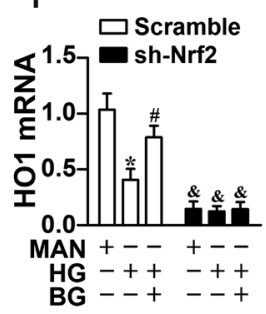

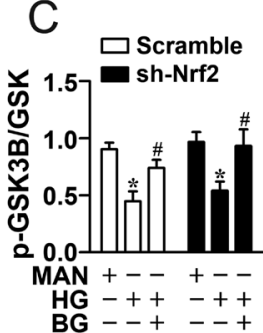

G

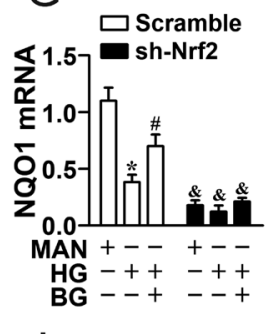

J

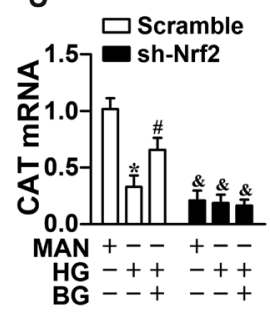

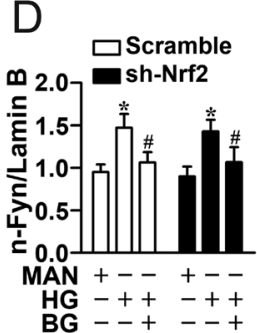

$\mathrm{H}$

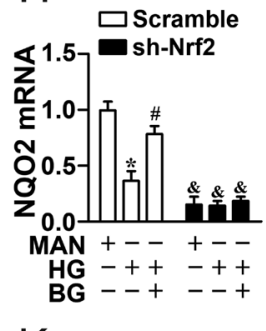

$\mathrm{K}$

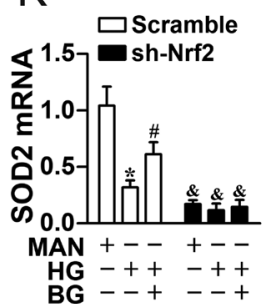

\section{Figure 9}

Nrf2 effects on the downstream of Akt/GSK3B/Fyn pathway. HUVECs were transduced with adenoviruses harboring sh-Nrf2 (Ad-sh-Nrf2) and a scrambled sequence (Ad-scramble) respectively. After transduction, HUVECs were cultured either in MAN or HG medium in the presence or absence of Baicalin for 72 h. (A, B, C, D and E) The protein expression and quantitative analysis of phosphorylation of Akt and GSK3B, the nuclear translocation of Fyn ( $\mathrm{n}-\mathrm{Fyn}$ ) and Nrf2 ( $n-N r f 2$ ) were evaluated by Western blot. The results were normalized to HUVECs transfected Ad-scramble expose to MAN, values displayed are means \pm S.E.M. of six independent experiments. * $P<0.05$ vs respective MAN in Ad-scramble-HUVECS or Ad-sh-Nrf2-HUVECs; $P<0.05$ vs respective HG in Ad-scramble-HUVECS or Ad-sh-Nrf2-HUVECs. (F, G, H, I, J and K) The mRNA expression and quantitation of Nrf2 downstream target genes were determined by sqRT-PCR. The results were normalized to HUVECs transfected Ad-scramble expose to MAN, values displayed are means \pm s.E.M. of six independent experiments. ${ }^{*} P<0.05$ vs HUVECs transfected Ad-scramble expose to MAN; $\# P<0.05$ vs HUVECs transfected Ad-scramble expose to HG; $\& P<0.05$ vs Ad-scramble-HUVECs with the same treatment.

The first innovative finding of this study is that the benefit of Baicalin requires activation of Nrf2 antioxidant signaling. Accumulating evidence demonstrates that oxidative stress in diabetes mellitus contributes to endothelial cell dysfunction (Dernbach et al. 2004, Sorrentino et al. 2007). Consistently, we found that the ROS and inflammation levels of HUVECs cultured with HG were increased, but significantly lowered by Baicalin, which indicates that Baicalin improves HUVECs antioxidant capacity and reduces pro-inflammatory cytokines such as IL6, IL8 and TNFa. In view of the fact that Nrf2 is the primary transcription factor, which functionally controls the antioxidant response essential for maintaining cellular redox homeostasis (Chan et al. 2001, Zhang 2006, Xue et al. 2008). Also as an adaptive mechanism, Nrf2 can be quickly upregulated in cells and tissues in response to various oxidative stresses, but downregulated in cells or tissue exposed to chronic oxidative stress (Suzuki et al. 2008, Cui et al. 2012). Corresponding with this view, we demonstrate for the first time that Baicalin markedly increased the nuclear accumulation of Nrf2 and the expression of downstream antioxidant target genes in this study, without altering total Nrf2 protein in HUVECs, which indicate that Baicalin improves HUVECs antioxidant capacity by activating Nrf2 transcriptional function and reduces the inflammation.

The essential role of Nrf2 plays in endothelial function has been widely appreciated. Florczyk et al. (2014) reported that lack of Nrf2 attenuated survival, proliferation and angiogenic functions of endothelial cells in vitro and in vivo. Furthermore, knockdown of Nrf2 inhibits angiogenesis of rat cardiac microvascular endothelial cells under hypoxic conditions (Kuang et al. 2013). Consistent with these findings, our studies revealed 
that adenoviral-mediated shRNA knockdown of Nrf2 substantially abrogated the protection effect of Baicalin on HUVECs survival, tube formation and anti-oxidative function in vitro. Moreover, ML385 almost abolished the ability of Baicalin to enhance angiogenesis ex vivo and the protective effects of Baicalin on vascular endothelium function in vivo and ex vivo. These findings revealed that Nrf2 plays an essential role in the ability of Baicalin to ameliorate HUVECs function.

The second important new finding is that the Akt/ GSK3B/Fyn pathway mediates the activation of Nrf2 induced by Baicalin in endothelial cells. The mechanism of Nrf2 activation has been widely investigated (Um et al. 2011, Bryan et al. 2013). In PC12 cells, it was found that puerarin triggers Akt activation, which in turn inhibits GSK3B activation and resulting in more Nrf2 nuclear translocation. Moreover, it was demonstrated that Baicalin attenuates ketamine-induced neurotoxicity in the developing rats (Zuo et al. 2016) and regulates antioxidant enzyme gene expression (Kim et al. 2012) by PI3K/Akt signaling. Consistently, we found that the decreased Akt phosphorylation induced by HG could be attenuated by Baicalin, which suggested that Baicalin could activate Akt in HUVECs and HAOECs. However, the direct linkage between the Baicalin and phosphorylation of Akt in HUVECs and HAOECs remains unknown.

The results from this work and other studies suggested that the Akt/GSK3B/Fyn pathway plays a pivotal role in regulation of Nrf2 nuclear export and degradation (Krakauer et al. 2001, Jain \& Jaiswal 2007, Jyrkkänen et al. 2008, Li et al. 2014). Based on the antioxidant effect of Baicalin-mediated by Akt activation, we hypothesized that Baicalin activates Nrf2 via the Akt/GSK3B/Fyn pathway.

Consistent with the hypothesis, we found that Baicalin not only increased the phosphorylation of Akt, but also inhibited GSK3B-mediated nuclear accumulation of Fyn. As Fyn is an Nrf2 negative regulator by entering into nuclei to phosphorylate Nrf2 tyrosines, subsequently promoting Nrf2 nuclear export and decreasing expression of its downstream genes (Jain \& Jaiswal 2007). Interestingly, however, there was no significant change in total Nrf2 protein level under any treatment condition in HUVECs, which demonstrate that the Baicalin promoted the nucleus enrichment of Nrf2 at macro level but not alter the balance between the total Nrf2 expression and its degradation. Importantly, we found that LY294002 almost completely abolished Baicalin-mediated activation of Nrf2 and expression of its downstream genes, accompanied with great attenuation of the protective effects of Baicalin on HUVECs survival and angiogenic function under HG treatment.

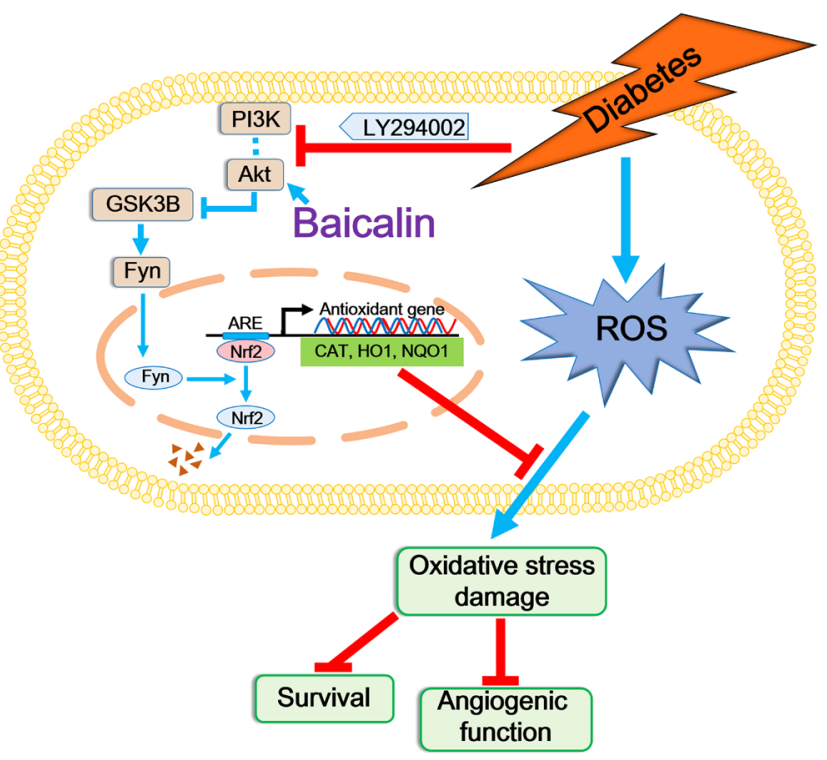

Figure 10

Baicalin alleviates hyperglycemia-induced endothelial impairment by downregulating oxidative damage via Nrf2 pathway. Schematic illustration of the protective effects of Baicalin on HUVECS under HG conditions. HG decreases expression of Nrf2 in HUVECs and induces oxidative stress, which impairs the survival and angiogenic function of HUVECs. Under HG conditions co-treatment with Baicalin improves HUVECs survival and function predominantly by Nrf2 activation mediated by increasing phosphorylation of Akt and GSK3B and inhibiting Fynmediated export of nuclear Nrf2. CAT indicates catalase; HO1, heme oxygenase 1; NQO1, NAD(P)H dehydrogenase (quinone 1); ROS, reactive oxygen species. A full color version of this figure is available at https://doi. org/10.1530/JOE-18-0457.

In conclusion, Baicalin improves HUVECs survival and function under HG conditions. This is predominantly because of increased Nrf2 activation, mediated in HUVECs by increasing Akt and GSK3B phosphorylation and inhibiting Fyn-mediated Nrf2 nuclear export. By preserving Nrf2 nuclear localization, Nrf2 function is enhanced and antioxidant gene expression is then increased, which in turn protecting HUVECs from hyperglycemia-induced oxidative damage (Fig. 10).

In summary, our findings indicate that Baicalin exerts vascular protective effect against hyperglycemia-induced ROS via AKT/GSK3B/Fyn-mediated Nrf2 activation in vivo and in vitro. Therefore, the results from the current study establish a novel role of Baicalin in endothelial protection, a finding that may have implications for the pathogenesis and treatment of diabetes-associated vascular complications.

\section{Declaration of interest}

The authors declare that there is no conflict of interest that could be perceived as prejudicing the impartiality of the research reported. 


\section{Funding}

This work was supported by grants from the National Natural Science Foundation of China $(81573069,81570368,81773346,81770498$ and 81800236) and the Natural Science Foundation of Zhejiang Province (LQ18H020004 and LYY18H310009).

\section{Author contribution statement}

Gen Chen, Xiangjuan Chen, Weitao Cong and Litai Jin conceived, designed and supervised the study and analyzed the data. Gen Chen, Xiaozhong Huang, Ning An and Shuai Huang performed experiments. Weijian Ye and Jia Sun interpreted data and reviewed the experimental settings. Xiangjuan Chen, Chao Niu and Santie Li contributed with intellectual expertise. Gen Chen and Yingjie Shen wrote the manuscript. Gen Chen, Shuai Huang and Jiaojiao Liang are the guarantors of this work and, as such, had full access to all the data in the study and take responsibility for the integrity of the data and the accuracy of the data analysis. All authors contributed to reviewing and editing the manuscript and approved its final version.

\section{References}

Aplin AC, Fogel E, Zorzi P \& Nicosia RF 2008 The aortic ring model of angiogenesis. Methods in Enzymology 443 119-136. (https://doi. org/10.1016/S0076-6879(08)02007-7)

Baker M, Robinson SD, Lechertier T, Barber PR, Tavora B, D'Amico G, Jones DT, Vojnovic B \& Hodivala-Dilke K 2011 Use of the mouse aortic ring assay to study angiogenesis. Nature Protocols 7 89-104. (https://doi.org/10.1038/nprot.2011.435)

Bhatt MP, Lee YJ, Jung SH, Kim YH, Hwang JY, Han ET, Park WS, Hong SH, Kim YM \& Ha KS 2016 C-peptide protects against hyperglycemic memory and vascular endothelial cell apoptosis. Journal of Endocrinology 231 97-108. (https://doi.org/10.1530/JOE-16-0349)

Brill A, Elinav H \& Varon D 2004 Differential role of platelet granular mediators in angiogenesis. Cardiovascular Research 63 226-235. (https://doi.org/10.1016/j.cardiores.2004.04.012)

Bryan HK, Olayanju A, Goldring CE \& Park BK 2013 The Nrf2 cell defence pathway: Keap1-dependent and -independent mechanisms of regulation. Biochemical Pharmacology 85 705-717. (https://doi. org/10.1016/j.bcp.2012.11.016)

Chan K, Han XD \& Kan YW 2001 An important function of Nrf2 in combating oxidative stress: detoxification of acetaminophen. PNAS 98 4611-4616. (https://doi.org/10.1073/pnas.081082098)

Chen WC, Kuo TH, Tzeng YS \& Tsai YC 2012 Baicalin induces apoptosis in SW620 human colorectal carcinoma cells in vitro and suppresses tumor growth in vivo. Molecules 17 3844-3857. (https://doi. org/10.3390/molecules17043844)

Cho NH, Shaw JE, Karuranga S, Huang Y, da Rocha Fernandes JD, Ohlrogge AW \& Malanda B 2018 IDF Diabetes Atlas: Global estimates of diabetes prevalence for 2017 and projections for 2045. Diabetes Research and Clinical Practice 138 271-281. (https://doi.org/10.1016/j. diabres.2018.02.023)

Cui W, Bai Y, Miao X, Luo P, Chen Q, Tan Y, Rane MJ, Miao L \& Cai L 2012 Prevention of diabetic nephropathy by sulforaphane: possible role of nrf2 upregulation and activation. Oxidative Medicine and Cellular Longevity 2012 821936. (https://doi. org/10.1155/2012/821936)

D'Autreaux B \& Toledano MB 2007 ROS as signalling molecules: mechanisms that generate specificity in ROS homeostasis. Nature Reviews Molecular Cell Biology 8 813-824. (https://doi.org/10.1038/ nrm2256)

Dernbach E, Urbich C, Brandes RP, Hofmann WK, Zeiher AM \& Dimmeler S 2004 Antioxidative stress-associated genes in circulating progenitor cells: evidence for enhanced resistance against oxidative stress. Blood 104 3591-3597. (https://doi.org/10.1182/blood-2003-124103)

Erusalimsky JD 2009 Vascular endothelial senescence: from mechanisms to pathophysiology. Journal of Applied Physiology 106 326-332. (https://doi.org/10.1152/japplphysiol.91353.2008)

Fang P, Yu M, Zhang L, Wan D, Shi M, Zhu Y, Bo P \& Zhang Z 2017 Baicalin against obesity and insulin resistance through activation of AKT/AS160/ GLUT4 pathway. Molecular and Cellular Endocrinology 448 77-86. (https://doi.org/10.1016/j.mce.2017.03.027)

Florczyk U, Jazwa A, Maleszewska M, Mendel M, Szade K, Kozakowska M, Grochot-Przeczek A, Viscardi M, Czauderna S, Bukowska-Strakova K, et al. 2014 Nrf2 regulates angiogenesis: effect on endothelial cells, bone marrow-derived proangiogenic cells and hind limb ischemia. Antioxidants and Redox Signaling 20 1693-1708. (https://doi. org/10.1089/ars.2013.5219)

Grundy SM, Howard B, Smith S Jr, Eckel R, Redberg R \& Bonow RO 2002 Prevention Conference VI: Diabetes and Cardiovascular Disease: executive summary: conference proceeding for healthcare professionals from a special writing group of the American Heart Association. Circulation 105 2231-2239. (https://doi.org/10.1161/01. CIR.0000013952.86046.DD)

Guzik TJ, Mossa S, Gastaldi D, Sadowski J, Ratnatunga C, Pilai R \& Channon KM 2002 Mechanisms of increased vascular superoxide production in human diabetes mellitus: role of $\mathrm{NAD}(\mathrm{P}) \mathrm{H}$ oxidase and endothelial nitric oxide synthase. Circulation 105 1656-1662. (https:// doi.org/10.1161/01.CIR.0000012748.58444.08)

Halliwell B, Rafter J \& Jenner A 2005 Health promotion by flavonoids, tocopherols, tocotrienols, and other phenols: direct or indirect effects? Antioxidant or not? American Journal of Clinical Nutrition $\mathbf{8 1}$ 268S-276S. (https://doi.org/10.1093/ajcn/81.1.268S)

Itoh K, Ishii T, Wakabayashi N \& Yamamoto M 1999 Regulatory mechanisms of cellular response to oxidative stress. Free Radical Research 31 319-324. (https://doi.org/10.1080/10715769900300881)

Jain AK \& Jaiswal AK 2007 GSK-3beta acts upstream of Fyn kinase in regulation of nuclear export and degradation of NF-E2 related factor 2. Journal of Biological Chemistry 282 16502-16510. (https://doi. org/10.1074/jbc.M611336200)

Jyrkkänen HK, Kansanen E, Inkala M, Kivelä AM, Hurttila H, Heinonen SE, Goldsteins G, Jauhiainen S, Tiainen S, Makkonen H, et al. $2008 \mathrm{Nrf2}$ regulates antioxidant gene expression evoked by oxidized phospholipids in endothelial cells and murine arteries in vivo. Circulation Research 103 e1-e9. (https://doi.org/10.1161/ CIRCRESAHA.108.176883)

Kim SH, Kim SH, Choi M, Lee Y, Kim YO, Ahn DS, Kim YH, Kang ES, Lee EJ, Jung M, et al. 2010 Natural therapeutic magnesium lithospermate B potently protects the endothelium from hyperglycaemia-induced dysfunction. Cardiovascular Research $\mathbf{8 7}$ 713-722. (https://doi.org/10.1093/cvr/cvq089)

Kim DH, Kim JM, Lee EK, Choi YJ, Kim CH, Choi JS, Kim ND, Yu BP \& Chung HY 2012 Modulation of FoxO1 phosphorylation/acetylation by baicalin during aging. Journal of Nutritional Biochemistry $\mathbf{2 3}$ 1277-1284. (https://doi.org/10.1016/j.jnutbio.2011.07.008)

Krakauer T, Li BQ \& Young HA 2001 The flavonoid Baicalin inhibits superantigen-induced inflammatory cytokines and chemokines. FEBS Letters 500 52-55. (https://doi.org/10.1016/S0014-5793(01)02584-4)

Kuang L, Feng J, He G \& Jing T 2013 Knockdown of Nrf2 inhibits the angiogenesis of rat cardiac micro-vascular endothelial cells under hypoxic conditions. International Journal of Biological Sciences 9 656-665. (https://doi.org/10.7150/ijbs.5887)

Li B, Liu S, Miao L \& Cai L 2012 Prevention of diabetic complications by activation of Nrf2: diabetic cardiomyopathy and nephropathy. Experimental Diabetes Research 2012 216512. (https://doi. org $/ 10.1155 / 2012 / 234084$ )

Li LF, Liu YY, Yang CT, Chien Y, Twu NF, Wang ML, Wang CY, Huang CC, Kao KC, Hsu HS, et al. 2013 Improvement of ventilator-induced lung injury by IPS cell-derived conditioned medium via inhibition of PI3K/ 
Akt pathway and IP-10-dependent paracrine regulation. Biomaterials 34 78-91. (https://doi.org/10.1016/j.biomaterials.2012.09.042)

Li C, Lin G \& Zuo Z 2011 Pharmacological effects and pharmacokinetics properties of Radix Scutellariae and its bioactive flavones. Biopharmaceutics and Drug Disposition 32 427-445. (https://doi. org/10.1002/bdd.771)

Li C, Pan Z, Xu T, Zhang C, Wu Q \& Niu Y 2014 Puerarin induces the upregulation of glutathione levels and nuclear translocation of Nrf2 through PI3K/Akt/GSK-3 $\beta$ signaling events in PC12 cells exposed to lead. Neurotoxicology and Teratology 46 1-9. (https://doi.org/10.1016/j. ntt.2014.08.007)

Li HT, Wu XD, Davey AK \& Wang J 2011 Antihyperglycemic effects of baicalin on streptozotocin-nicotinamide induced diabetic rats. Phytotherapy Research 25 189-194. (https://doi.org/10.1002/ptr.3433)

Li H, Zhang L, Wang F, Shi Y, Ren Y, Liu Q, Cao Y \& Duan H 2011 Attenuation of glomerular injury in diabetic mice with tertbutylhydroquinone through nuclear factor erythroid 2-related factor 2-dependent antioxidant gene activation. American Journal of Nephrology 33 289-297. (https://doi.org/10.1159/000324694)

Lin Y, Berg AH, Iyengar P, Lam TK, Giacca A, Combs TP, Rajala MW, Du X, Rollman B, Li W, et al. 2005 The hyperglycemia-induced inflammatory response in adipocytes: the role of reactive oxygen species. Journal of Biological Chemistry 280 4617-4626. (https://doi. org/10.1074/jbc.M411863200)

Lin M, Li L, Zhang Y, Zheng L, Xu M, Rong R \& Zhu T 2014 Baicalin ameliorates $\mathrm{H}_{2} \mathrm{O}_{2}$ induced cytotoxicity in HK-2 cells through the inhibition of ER stress and the activation of Nrf2 signaling. International Journal of Molecular Sciences 15 12507-12522. (https:// doi.org/10.3390/ijms150712507)

Lu T, Chai Q, Yu L, d'Uscio LV, Katusic ZS, He T \& Lee HC 2012 Reactive oxygen species signaling facilitates FOXO-3 $\alpha / \mathrm{FBXO}$-dependent vascular BK channel $\beta 1$ subunit degradation in diabetic mice. Diabetes 61 1860-1868. (https://doi.org/10.2337/db11-1658)

Maejima Y, Kyoi S, Zhai P, Liu T, Li H, Ivessa A, Sciarretta S, Del Re DP, Zablocki DK \& Hsu CP 2013 Mst1 inhibits autophagy by promoting the interaction between Beclin1 and Bcl-2. Nature Medicine 19 1478-1488. (https://doi.org/10.1038/nm.3322)

Magalhães J, Falcão-Pires I, Gonçalves IO, Lumini-Oliveira J, Marques-Aleixo I, Dos Passos E, Rocha-Rodrigues S, Machado NG, Moreira AC \& Miranda-Silva D 2013 Synergistic impact of endurance training and intermittent hypobaric hypoxia on cardiac function and mitochondrial energetic and signaling. International Journal of Cardiology 168 5363-5371. (https://doi.org/10.1016/j. ijcard.2013.08.001)

Palsamy P \& Subramanian S 2011 Resveratrol protects diabetic kidney by attenuating hyperglycemia-mediated oxidative stress and renal inflammatory cytokines via Nrf2-Keap1 signaling. Biochimica et Biophysica Acta 1812 719-731. (https://doi.org/10.1016/j. bbadis.2011.03.008)

Pitocco D, Tesauro M, Alessandro R, Ghirlanda G \& Cardillo C 2013 Oxidative stress in diabetes: implications for vascular and other complications. International Journal of Molecular Sciences $\mathbf{1 4}$ 21525-21550. (https://doi.org/10.3390/ijms141121525)

Rask-Madsen C \& King GL 2013 Vascular complications of diabetes: mechanisms of injury and protective factors. Cell Metabolism $\mathbf{1 7}$ 20-33. (https://doi.org/10.1016/j.cmet.2012.11.012)

Singh A, Venkannagari S, Oh KH, Zhang YQ, Rohde JM, Liu L, Nimmagadda S, Sudini K, Brimacombe KR, Gajghate S, et al. 2016 Small molecule inhibitor of NRF2 selectively intervenes therapeutic resistance in KEAP1-deficient NSCLC tumors. ACS Chemical Biology 11 3214-3225. (https://doi.org/10.1021/acschembio.6b00651)
Sorrentino SA, Bahlmann FH, Besler C, Müller M, Schulz S, Kirchhoff N, Doerries C, Horváth T, Limbourg A, Limbourg F, et al. 2007 Oxidant stress impairs in vivo reendothelialization capacity of endothelial progenitor cells from patients with type 2 diabetes mellitus: restoration by the peroxisome proliferator-activated receptorgamma agonist rosiglitazone. Circulation 116 163-173. (https://doi. org/10.1161/CIRCULATIONAHA.106.684381)

Srinivas NR 2010 Baicalin, an emerging multi-therapeutic agent: pharmacodynamics, pharmacokinetics, and considerations from drug development perspectives. Xenobiotica 40 357-367. (https://doi. org/10.3109/00498251003663724)

Suzuki M, Betsuyaku T, Ito Y, Nagai K, Nasuhara Y, Kaga K, Kondo S \& Nishimura M 2008 Down-regulated NF-E2-related factor 2 in pulmonary macrophages of aged smokers and patients with chronic obstructive pulmonary disease. American Journal of Respiratory Cell and Molecular Biology 39 673-682. (https://doi.org/10.1165/rcmb.2007-0424OC)

Tang SC, Liao PY, Hung SJ, Ge JS, Chen SM, Lai JC, Hsiao YP \& Yang JH 2017 Topical application of glycolic acid suppresses the UVB induced IL-6, IL-8, MCP-1 and COX-2 inflammation by modulating NF-kB signaling pathway in keratinocytes and mice skin. Journal of Dermatological Science 86 238-248. (https://doi.org/10.1016/j. jdermsci.2017.03.004)

Um HC, Jang JH, Kim DH, Lee C \& Surh YJ 2011 Nitric oxide activates Nrf2 through S-nitrosylation of Keap1 in PC12 cells. Nitric Oxide 25 161-168. (https://doi.org/10.1016/j.niox.2011.06.001)

Vallée A \& Lecarpentier Y 2018 Crosstalk between peroxisome proliferator-activated receptor gamma and the canonical WNT/ $\beta$ Catenin pathway in chronic inflammation and oxidative stress during carcinogenesis. Frontiers in Immunology 9 745. (https://doi. org/10.3389/fimmu.2018.00745)

Waisundara VY, Siu SY, Hsu A, Huang D \& Tan BK 2011 Baicalin upregulates the genetic expression of antioxidant enzymes in Type-2 diabetic Goto-Kakizaki rats. Life Sciences 88 1016-1025. (https://doi. org/10.1016/j.lfs.2011.03.009)

Wang G, Liang J, Gao LR, Si ZP, Zhang XT, Liang G, Yan Y, Li K, Cheng X, Bao Y, et al. 2018 Baicalin administration attenuates hyperglycemiainduced malformation of cardiovascular system. Cell Death and Disease 9 234. (https://doi.org/10.1038/s41419-018-0318-2)

Williamson G, Barron D, Shimoi K \& Terao J 2005 In vitro biological properties of flavonoid conjugates found in vivo. Free Radical Research 39 457-469. (https://doi.org/10.1080/10715760500053610)

Xie Z, Zhang J, Wu J, Viollet B \& Zou MH 2008 upregulation of mitochondrial uncoupling protein- 2 by the AMP-activated protein kinase in endothelial cells attenuates oxidative stress in diabetes. Diabetes 57 3222-3230. (https://doi.org/10.2337/db08-0610)

Xue M, Qian Q, Adaikalakoteswari A, Rabbani N, Babaei-Jadidi R $\&$ Thornalley PJ 2008 Activation of NF-E2-related factor-2 reverses biochemical dysfunction of endothelial cells induced by hyperglycemia linked to vascular disease. Diabetes 57 2809-2817. (https://doi.org/10.2337/db06-1003)

Zhang DD 2006 Mechanistic studies of the Nrf2-Keap1 signaling pathway. Drug Metabolism Reviews 38 769-789. (https://doi. org/10.1080/03602530600971974)

Zou C, Wang Y \& Shen Z 2005 2-NBDG as a fluorescent indicator for direct glucose uptake measurement. Journal of Biochemical and Biophysical Methods 64 207-215. (https://doi.org/10.1016/j. jbbm.2005.08.001)

Zuo D, Lin L, Liu Y, Wang C, Xu J, Sun F, Li L, Li Z \& Wu Y 2016 Baicalin attenuates ketamine-induced neurotoxicity in the developing rats: involvement of PI3K/Akt and CREB/BDNF/Bcl-2 pathways. Neurotoxicity Research 30 159-172. (https://doi.org/10.1007/s12640-016-9611-y) https://joe.bioscientifica.com

https://doi.org/10.1530/JOE-18-0457 (c) 2019 Society for Endocrinology Published by Bioscientifica Ltd. Printed in Great Britain
Received in final form 25 September 2018

Accepted 4 October 2018

Accepted Preprint published online 4 October 2018 